\title{
\begin{tabular}{l|l} 
Mibraries & DSpace@MIT
\end{tabular}
}

MIT Open Access Articles

Surface Water Dependent Properties of Sulfur-Rich
Molybdenum Sulfides: Electrolyteless Gas Phase Water Splitting

The MIT Faculty has made this article openly available. Please share how this access benefits you. Your story matters.

As Published: 10.1021/ACSNANO.7B01632

Publisher: American Chemical Society (ACS)

Persistent URL: https://hdl.handle.net/1721.1/133922

Version: Final published version: final published article, as it appeared in a journal, conference proceedings, or other formally published context

Terms of Use: Article is made available in accordance with the publisher's policy and may be subject to US copyright law. Please refer to the publisher's site for terms of use. 


\section{Surface Water Dependent Properties of Sulfur- Rich Molybdenum Sulfides: Electrolyteless Gas Phase Water Splitting}

Torben Daeneke, ${ }^{*}, \dagger, \perp \odot$ Nripen Dahr, ${ }^{\dagger, \perp}$ Paul Atkin, ${ }^{\dagger}$ Rhiannon M. Clark, ${ }^{\dagger}$ Christopher J. Harrison, ${ }^{\dagger}$ Robert Brkljača, ${ }^{\ddagger}$ Naresh Pillai, ${ }^{\dagger}$ Bao Yue Zhang, ${ }^{\dagger}$ Ali Zavabeti, ${ }^{\dagger}$ Samuel J. Ippolito, ${ }^{\dagger}$ Kyle J. Berean, ${ }^{\dagger}$ Jian Zhen Ou, ${ }^{\dagger}$ Michael S. Strano, ${ }^{\S \odot}$ and Kourosh Kalantar-zadeh*,†

${ }^{\dagger}$ School of Engineering and ${ }^{\ddagger}$ School of Science, RMIT University, 124 La Trobe Street, 3001 Melbourne, Victoria, Australia

${ }^{\S}$ Department of Chemical Engineering, Massachusetts Institute of Technology, 77 Massachusetts Avenue, 02139 Cambridge,

Massachusetts, United States

Supporting Information

ABSTRACT: Sulfur-rich molybdenum sulfides are an emerging class of inorganic coordination polymers that are predominantly utilized for their superior catalytic properties. Here we investigate surface water dependent properties of sulfur-rich $\operatorname{MoS}_{x}\left(x=3^{2} / 3\right)$ and its interaction with water vapor. We report that $M_{0} S_{x}$ is a highly hygroscopic semiconductor, which can reversibly bind up to $0.9 \mathrm{H}_{2} \mathrm{O}$ molecule per Mo. The presence of surface water is found to have a profound influence on the semiconductor's properties, modulating the material's photoluminescence by over 1 order of magnitude, in transition from dry to moist ambient. Furthermore, the

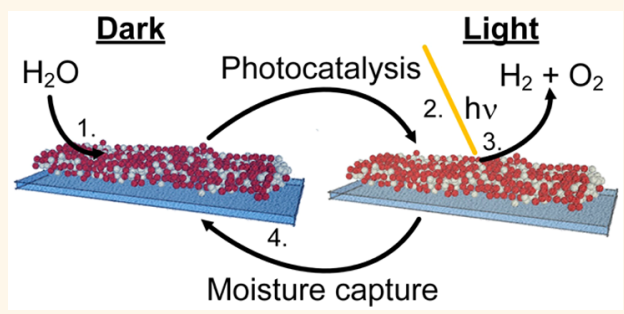
conductivity of a $\mathrm{MoS}_{x}$-based moisture sensor is modulated in excess of 2 orders of magnitude for $30 \%$ increase in humidity. As the core application, we utilize the discovered properties of $\mathrm{MoS}_{x}$ to develop an electrolyteless water splitting photocatalyst that relies entirely on the hygroscopic nature of $M_{x} S_{x}$ as the water source. The catalyst is formulated as an ink that can be coated onto insulating substrates, such as glass, leading to efficient hydrogen and oxygen evolution from water vapor. The concept has the potential to be widely adopted for future solar fuel production.

KEYWORDS: electrolyteless water splitting, amorphous molybdenum sulfide, photocatalysis, hydrogen production, MoS moisture sensing, desiccation

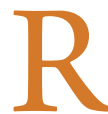

enewable hydrogen has the potential to transition the current fossil fuel based economy and infrastructure to incorporate a larger share of carbon-neutral clean fuel. Hydrogen production utilizing water electrolysis or direct photocatalytic splitting of water is currently regarded as the most common pathway toward a hydrogen-based economy. ${ }^{1,2}$ Therefore, the vast majority of research on hydrogen evolution catalysis is conducted using liquid phase electrolytes. However, the utilization of liquid electrolytes has intrinsic efficiencylimiting and cost-increasing disadvantages that arise from issues including bubble formation, freezing at low temperatures, the necessity of liquid pumping, corrosion, and catalyst poisoning. ${ }^{3}$ In contrast, by resorting to gas phase water splitting these shortcomings can be surmounted. Advantageously, gas phase water splitting is predicted to require less energy, due to the lower standard Gibbs free energy of formation of gaseous water, $\Delta G_{\mathrm{f}}^{0}\left[\mathrm{H}_{2} \mathrm{O}(\mathrm{g})\right]=-228.59 \mathrm{~kJ} \mathrm{~mol}^{-1}$, compared to liquid water, $\Delta G_{\mathrm{f}}^{0}\left[\mathrm{H}_{2} \mathrm{O}(\mathrm{l})\right]=-237.18 \mathrm{~kJ} \mathrm{~mol}^{-1}$. To date, only a few gas phase water splitting systems have been reported. ${ }^{3,5,6}$ However, their hydrogen evolution rates still lag behind the ones obtained following the traditional liquid electrolyte based approaches, and they also generally require the fabrication of complex microfluidic devices, negating many of the potential advantages.

Herein we report a high-efficiency vapor phase photocatalytic water splitting system, which can be formulated as an ink, allowing adapting any surface for gas phase hydrogen fuel production, drastically simplifying the hydrogen evolution system. The development of this semiconducting moisture absorber is highly relevant to the field of hydrogen evolution catalysis, while also finding other applications such as resistive moisture sensing and dehumidification.

The ideal material for efficient electrolyteless gas phase water splitting applications should (I) be highly porous, to enable efficient fuel gas and water vapor transport, (II) feature a large moisture adsorption capacity for binding water molecules, (III) be a semiconductor with good conductivity, providing light adsorption capabilities, and (IV) feature high catalytic activity.

Received: March 7, 2017

Accepted: May 30, 2017

Published: June 14, 2017 
a)

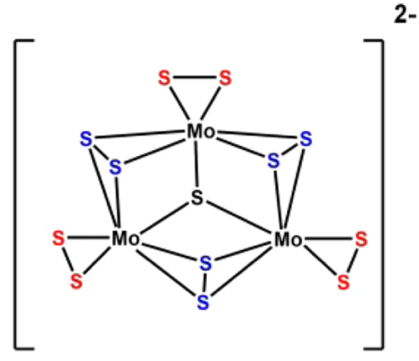

b)

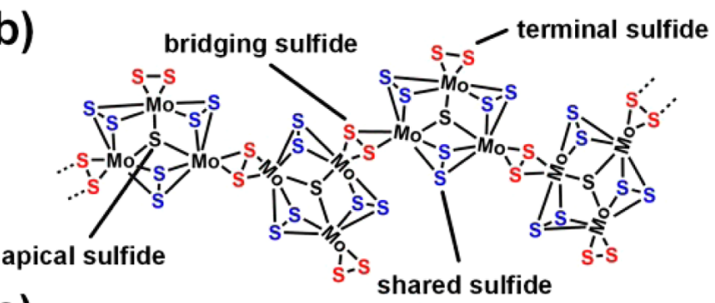

c)

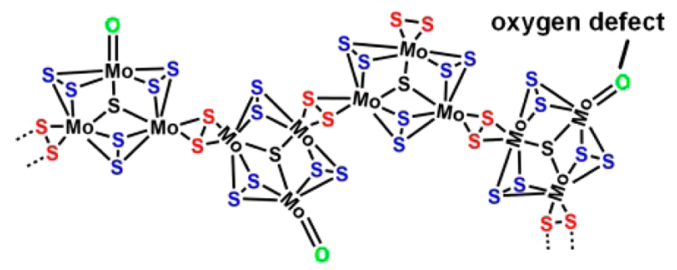

d)

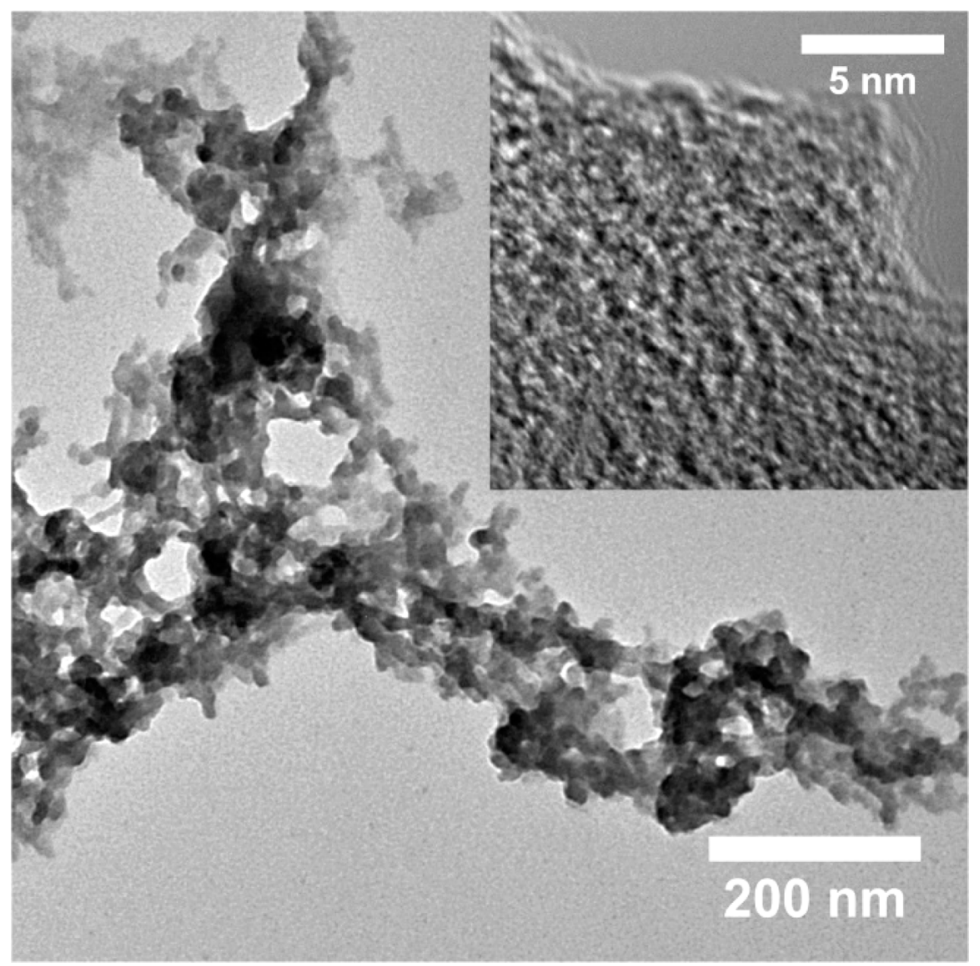

Figure 1. Chemical structure and morphology of $\mathrm{MoS}_{x}$ : (a) chemical structure of $\mathrm{Mo}_{3} \mathrm{~S}_{13}{ }^{2-}$, (b) stoichiometric polymerized $\mathrm{MoS}_{3}{ }^{2} / 3$ with highlighted and color-coded sulfur moieties, (c) defected $M_{0} S_{x}$ featuring oxygen defects, (d) TEM image of the synthesized product and a HRTEM image (inset) showing ribbon-like features, forming due to the porous and branched nature of the compound.

Traditional efficient moisture-adsorbing materials, such as silica gel and zeolites, are insulators and hence not suitable for creating electrolyteless gas phase water splitting. Semiconducting organic polymers and metal organic frameworks can potentially feature the required electronic properties; however, their vulnerability to moisture-induced degradation remains a challenge. ${ }^{7}$ Furthermore, hydrocarbon-based materials lack the rich catalytic properties that are required. ${ }^{8,9}$ Moisture-adsorbing semiconducting clays and other stratified materials might be more suitable; ${ }^{10}$ however gas transport into the interlayer spacing is expected to be slow.

Transition metal based inorganic semiconducting polymers (TMISP) may be considered as candidates for electrolyteless gas phase water splitting. They can offer catalytic properties, which arise from their partially filled d-shells. Fortunately, many TMISPs are recognized for their catalytic properties, but their moisture-binding capabilities have not been investigated. ${ }^{11,12}$ Our study focuses on sulfur-rich amorphous molybdenum sulfide $\left(\operatorname{MoS}_{x}\right)$, which has recently gained increasing attention due to its exceptional catalysis. This class of materials encompasses a range of compounds featuring molybdenum to sulfur ratios anywhere between 2 and $6 .^{13-20}$

$\mathrm{MoS}_{x}$-based materials are recognized as promising noble metal free water-splitting catalysts, offering hydrogen evolution process efficiencies approaching that of platinum. ${ }^{15,17,19-22}$ However, other than $\mathrm{MoS}_{2}$, there is still considerable debate over the structure of these materials and the nature of their catalytically active sites. Tran et al. recently reinvestigated the structure of the commonly used electrodeposited $\mathrm{MoS}_{x}$ catalysts, which were believed to be $\mathrm{MoS}_{3}$ until then. ${ }^{11}$ It was discovered that this material's stoichiometry is closer to $\mathrm{MoS}_{4}$, being an inorganic coordination polymer with the formula of $\mathrm{MoS}_{3}{ }^{2} /{ }^{2}$, consisting of the polymerized $\mathrm{Mo}_{3} \mathrm{~S}_{13}{ }^{-2}$ nanoclusters
(Figure 1a and b). Irrespective of the exact stoichiometry, many $\mathrm{MoS}_{x}$ compounds are made of quasi-one-dimensional and branched inorganic coordination polymers or their shorterchain oligomers and monomers. ${ }^{11,23}$

While initial investigations had suggested that the sulfur-rich molybdenum sulfides are precursors to nanometer-sized $\mathrm{MoS}_{2}$ crystals (another, albeit less efficient, hydrogen evolution catalysts), it is becoming increasingly clear that this is not the case. $^{11,12,21,24,25}$ Recent studies implicate either the terminal disulfide ligands or bridging disulfides as the catalytically active sites, while a consensus is yet to be reached (Figure $1 \mathrm{~b}$ and c). ${ }^{11,12,26,27}$

Most sulfur-rich $\mathrm{MoS}_{x}$ semiconductors feature narrow band gaps of approximately $1.2 \mathrm{eV}$ and hence show high conductivity. ${ }^{28}$ The high conductivity of $\mathrm{MoS}_{x}$ is considered a key feature enabling its exceptional performance as an electrocatalyst. ${ }^{19,29,30}$ The optical band gap of this material is however too narrow to achieve direct photocatalytic water splitting. ${ }^{31}$ One approach to overcoming this limitation is coating photoelectrodes consisting of semiconducting $\mathrm{CuO}$, p$\mathrm{Si}$, or organic semiconductors with $\mathrm{MoS}_{x}$ catalysts. ${ }^{32-38}$

While the main research effort into $\mathrm{MoS}_{x}$ is focused on the hydrogen evolution reaction, it has also been demonstrated to be an effective $\mathrm{CO}_{2}$ reduction catalyst. ${ }^{39}$ Sulfur-rich molybdenum sulfides have found further applications in capturing toxic iodine and mercury. ${ }^{40}$ Furthermore, the high polarizability of $\mathrm{MoS}_{x}$ allows the binding of polar gaseous contaminants, including $\mathrm{CO}_{2}$ and certain volatile organic compounds. ${ }^{40}$ Other applications include energy storage in supercapacitors and alkali ion batteries. ${ }^{41,42}$

Still relatively little is known about the interactions between water molecules and $\mathrm{MoS}_{x}$ or the effects that hydration has on $\mathrm{MoS}_{x}$ properties. In this work, we investigate the effect of water 
vapor on $\mathrm{MoS}_{3}{ }^{2} / 3$, specifically focusing on its influence on the material's electronic properties.

We also explore $\operatorname{MoS}_{x}$ for moisture sensing and dehumidification. Moisture sensors are typically made of oxides, due to the importance of terminal oxygen moieties for moisture sensing. ${ }^{43,44}$ The demonstration of TMISPs as moisture sensors introduces an additional class of materials for humidity sensing. Dehumidification of ambient air, particularly in large climatecontrolled buildings, leads to significant consumption of energy. ${ }^{45}$ Classical desiccants such as silica gel and zeolites are often regenerated at temperatures above $70{ }^{\circ} \mathrm{C} .4^{46,47}$ Reduction of the regeneration temperature, while maintaining fast desorption kinetics, may lead to significant energy savings. 45,48

Finally, electrolyteless photocatalytic water splitting is demonstrated only relying on moisture captured from humid air as a water source. For this purpose, a catalytic ink is developed that can be coated onto any substrate, leading to efficient and low-cost hydrogen production from humid environments.

\section{RESULTS AND DISCUSSION}

Synthesis and Characterization. Partially hydrated $\mathrm{MoS}_{x}$ was synthesized following a modified literature procedure. ${ }^{14,35}$ In short, $\mathrm{MoS}_{4}\left(\mathrm{NH}_{4}\right)_{2}$ was added to an aqueous solution of poly(allylamine hydrochloride) (PAAHCl). The cationic polymer acts as a templating backbone during the synthesis, binding the precursor and leading to preferential assembly of $\mathrm{MoS}_{4}{ }^{2-}$ ions along one dimension. The solution was then acidified using a $\mathrm{HCl}$ vapor acidification method, devised to gradually lower the $\mathrm{pH}$, leading to polymerization. The resulting product was then thoroughly washed to remove the remaining precursors and side products. Raman spectroscopy confirmed the successful removal of the polymer template (see Figure SI-1).

Transmission electron microscopy (TEM) revealed that a highly porous network of quasi-one-dimensional nanostructures was obtained (Figure 1d), consisting predominantly of 10-20 $\mathrm{nm}$ wide interconnected branches of $\mathrm{MoS}_{x}$. The material was observed to be prone to beam damage during high-resolution imaging (see Figure SI-2), which is known to induce sulfur loss, gradually converting $\operatorname{MoS}_{x}(x>2)$ to $\mathrm{MoS}_{2}{ }^{49}$ As such, care was taken to avoid beam damage during the following characterizations. As expected, high-resolution TEM (HRTEM) imaging confirmed that the product is amorphous, featuring no distinguishable lattice structures. On the basis of previously published reports, it is concluded that the synthesized $\operatorname{MoS}_{x}$ chains have agglomerated, forming 10-20 nm wide bundles, which are interconnected to establish a highly porous superstructure. ${ }^{11,50}$ X-ray diffraction (XRD) confirmed the amorphous nature of the product (Figure SI-3). ${ }^{14}$ The addition of the PAAHCl template was found to increase porosity and reduce feature sizes, when compared to $\mathrm{MoS}_{x}$ synthesized without any scaffold (Figure SI-4). The observed morphology is in good agreement with previous reports. ${ }^{14,35}$

The absorption band gap of the synthesized material was determined to be $1.22 \mathrm{eV}$, using UV-vis spectroscopy, which is close to the reported value of $1.25 \mathrm{eV}$ (Figure 2a). ${ }^{28}$ Furthermore, an additional absorption feature was observed close to the absorption onset at $963 \mathrm{~nm}$ (Figure 2a). This feature has been identified as the Urbach tail, which is frequently observed in amorphous semiconductors. ${ }^{28} \mathrm{X}$-ray
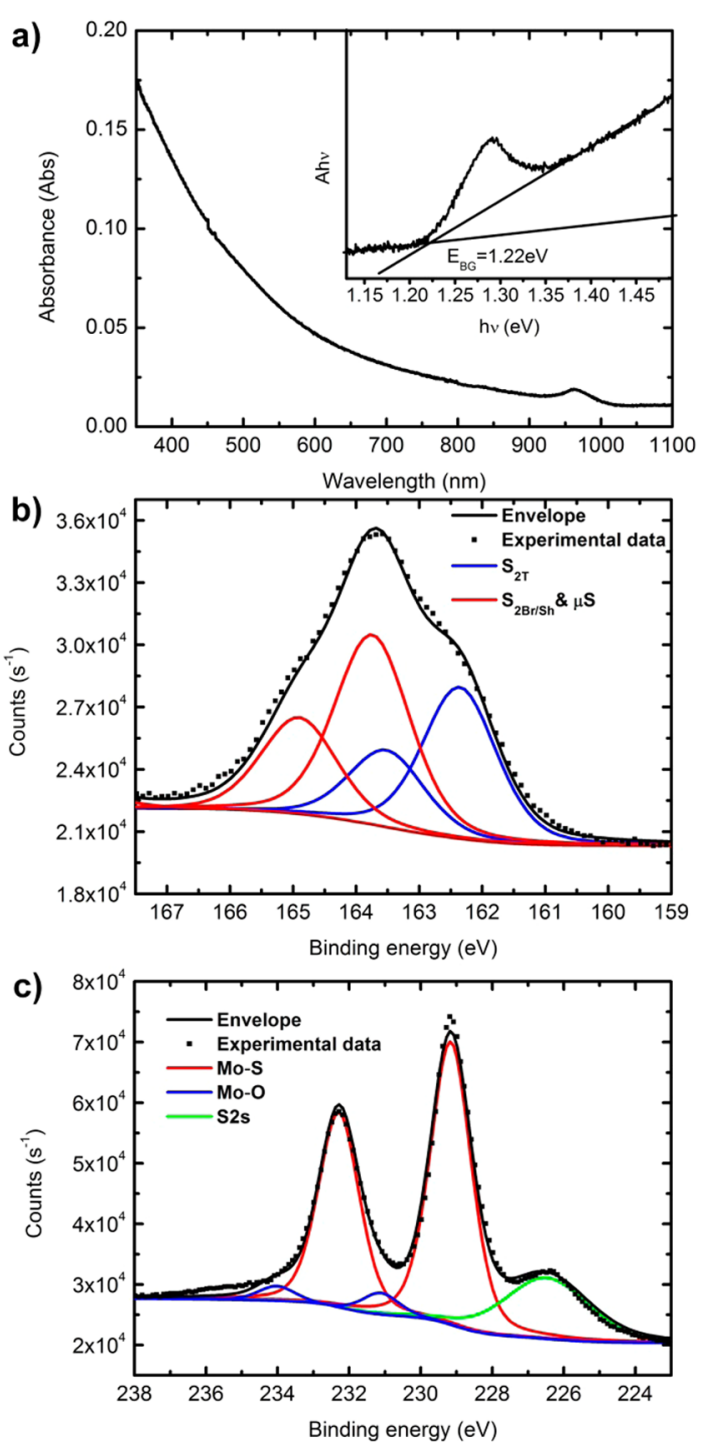

Figure 2. Assessing the band gap and stoichiometry of $\operatorname{MoS}_{x}$ : (a) UV-vis spectrum obtained in aqueous suspension and the corresponding Tauc plot (inset), (b) XPS spectrum of the sulfur $2 p$ region, and (c) XPS spectrum of the molybdenum $3 d$ region. In the sulfur $2 p$ region two overlapping doublets are observed, which are associated with terminal disulfide ligands $\left(S_{2 \mathrm{~T}}\right)(162.3$ and $163.5 \mathrm{eV})$ and bridging or shared disulfide ligands $\left(\mathrm{S}_{2 \mathrm{Br} / \mathrm{Sh}}\right)(163.7$ and $164.9 \mathrm{eV}$ ) (Figure $1 \mathrm{a}$ and $\mathrm{b}) .{ }^{11,23}$ The close proximity of the $S_{2 \mathrm{Br} / \mathrm{Sh}}-\mathrm{S} 2 \mathrm{p}_{3 / 2}$ and the $S_{2 \mathrm{~T}}-\mathrm{S} 2 \mathrm{p}_{1 / 2}$ peaks leads to the characteristic triplet-like appearance of the XPS signal $(b)$. The molybdenum $3 \mathrm{~d}$ region features two distinct doublets and one singlet (c). The singlet arises due to the sulfur S2 signal. Its broad nature is observed due to the presence of sulfur atoms in various environments and oxidation states. ${ }^{23}$ The lower energy doublet corresponds to molybdenum-sulfur bonds, while the higher energy doublet is associated with molybdenum-oxygen bonds. ${ }^{11}$ The presence of oxygen defects is commonly observed in $\mathrm{MoS}_{3}{ }^{2} / 3$, and the structure of defected $\mathrm{MoS}_{3{ }^{2} / 3}$ is shown in Figure 1c. ${ }^{11}$

photoelectron spectroscopy (XPS) was then employed to obtain the composition of the product, attesting a sulfur to molybdenum ratio of $78 \%$ to $22 \%$, which indicates a stoichiometry close to $\mathrm{MoS}_{3}{ }^{2} / 3$. The XPS spectrum of the material shows the expected features in the sulfur and molybdenum regions (Figure 2). ${ }^{11}$ 
Hygroscopic Properties and Low-Energy Dehumidification. Due to the highly porous structure of the network of the synthesized $\mathrm{MoS}_{3}{ }^{2} / 3$ sample and the known affinity of amorphous molybdenum sulfides toward polarizable gases, we predict that the material should feature intense interaction with gaseous substances including water vapor. Hence we decided to investigate the effects of moisture adsorption on the electronic properties of the material. ${ }^{40}$

Moisture desorption isotherms were recorded under nitrogen flow at set temperatures in a thermogravimetric analyzer in order to quantify the moisture capacity of amorphous $\mathrm{MoS}_{3}{ }^{2} / 3$. Weight loss curves were recorded at set temperatures (Figure 3a), which revealed that the material contained a significant weight fraction of labile water. A $7 \%$ weight loss could be achieved even at moderate temperatures of $\geq 40{ }^{\circ} \mathrm{C}$. The weight
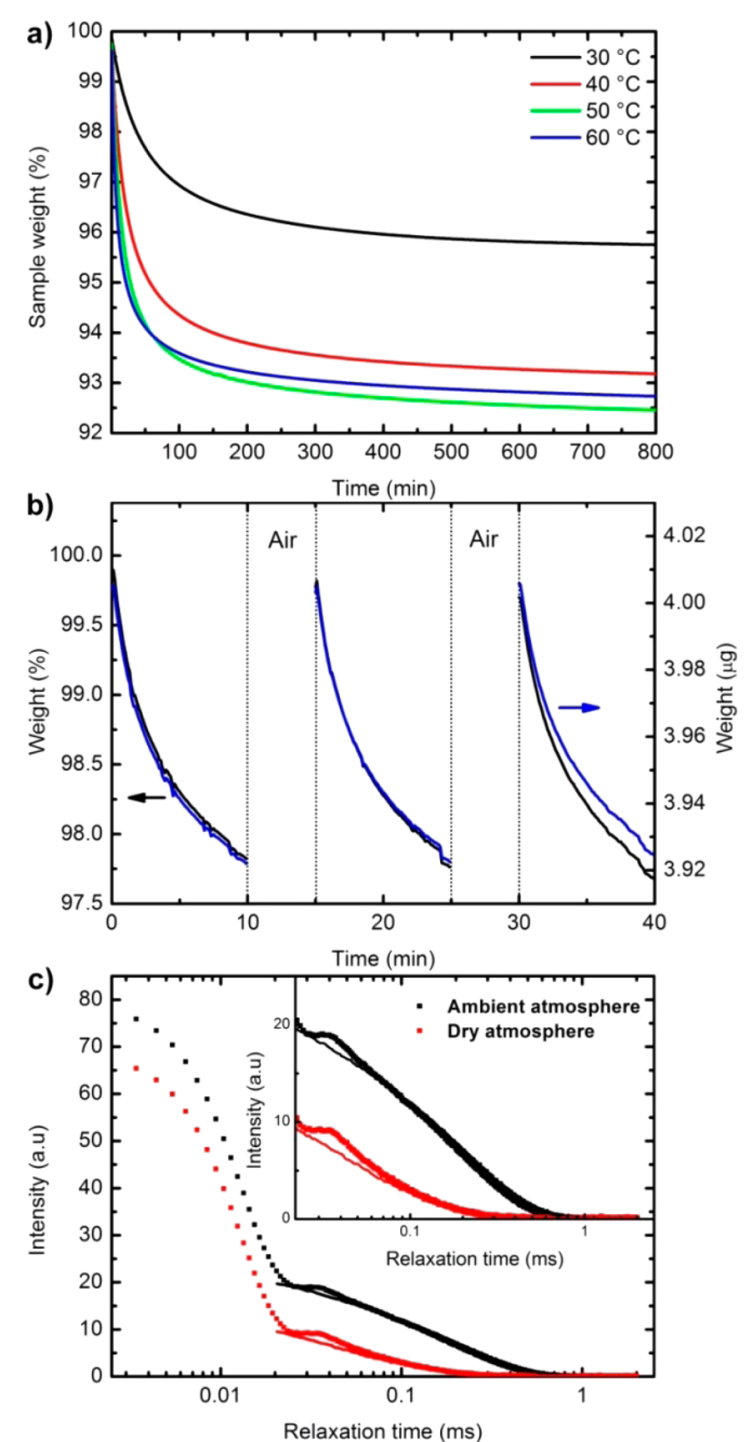

Figure 3. Assessment of the moisture uptake by $\mathrm{MoS}_{3{ }^{2} / 3}$ : (a) moisture desorption curves recorded at indicated temperatures, (b) cyclic moisture desorption and adsorption, in which desorption occurred in $40{ }^{\circ} \mathrm{C}$ dry nitrogen $(10 \mathrm{~min})$ and adsorption occurred at room temperature in ambient atmosphere (relative humidity controlled to $50 \% ; 5 \mathrm{~min}$ ), (c) solid-echo low-field NMR relaxation data (squares) and spin-echo low-field NMR relaxation data (solid lines); inset: magnified plot of the labile water region. loss occurred rapidly upon exposure to dry atmosphere, attesting fast desorption kinetics. Furthermore, we observed that the dried material quickly readsorbed the lost moisture when exposed to ambient atmosphere, highlighting the reversible nature of the reaction. Figure $3 \mathrm{~b}$ showcases a cyclic adsorption and desorption experiment at $40{ }^{\circ} \mathrm{C}$ in which the sample was exposed to dry nitrogen followed by exposure to ambient atmosphere. It can be observed that the $\mathrm{MoS}_{3}{ }^{2} / 3$ sample rapidly loses $2 \%$ of its weight in dry nitrogen, which is then reabsorbed from ambient air within $5 \mathrm{~min}$. This process could be repeated several times, indicating that the moisture adsorption and desorption were highly reversible, and the sample weight returned to its original value following each cycle. This behavior indicates that the $\mathrm{H}_{2} \mathrm{O}$ molecules are bound through relatively weak physisorption processes. The observed properties show that $\mathrm{MoS}_{3}{ }^{2} / 3$ is a suitable desiccant, capable of rapidly adsorbing and removing moisture from humid air, which can function in a comparatively low temperature regeneration step. While the moisture capacity is lower than that of state-of-the-art silica gel and zeolite-based desiccants, which can typically accommodate $20 \%$ to $40 \%$ of water by weight, regeneration can occur at comparatively lower temperatures, which render $\mathrm{MoS}_{3 / 3}{ }^{2}$ as an attractive alternative for continuous low-energy moisture removal. ${ }^{51}$

Low-field NMR relaxation studies were carried out to gain further insight into the moisture-binding process observed in partially hydrated $\mathrm{MoS}_{3}{ }^{2} / 3$. The measured spin-spin relaxation times $\left(T_{2}\right)$ provide insight into the environment of the respective water molecules. Crystalline water, firmly bound into the lattice, features relaxation times on the order of $10^{-6}$ to $10^{-5} \mathrm{~s}$, while weakly bound, labile water leads to relaxation times on the order of $10^{-4} \mathrm{~s} .{ }^{52}$ Even longer relaxation times exceeding milliseconds are observed for liquid water. Here, relaxation time measurements were conducted using solidecho and spin-echo protocols. ${ }^{53,54}$ The solid-echo protocol is optimized to accurately determine relaxation times on the order of microseconds, while the spin-echo measurement is suitable for assessing longer relaxation times. For these experiments samples stored in ambient atmosphere and dry atmosphere were compared (Figure 3c). The data were fitted in order to retrieve the $T_{2}$ relaxation times (Table SI- 1 ).

On visually inspecting the solid-echo and spin-echo NMR data (Figure 3c), it can be seen that the signal from both samples is similar in the short time scale, while significant changes occurred for longer time scales. The fit to the solidecho data revealed that the water content within the ambient atmosphere sample consisted of approximately $74 \%$ crystalline water with the remainder being loosely adsorbed and labile water. This labile water is likely bound by van der Waals interactions and hydrogen bonds (physisorption), while the crystalline component is likely associated with the water of crystallization, which is more tightly bound inside the material through coordination. Upon drying, this ratio changed to $84 \%$ crystalline water with only $16 \%$ labile water, confirming that a significant fraction of water has been lost. The spin-echo measurements provide a more accurate measure of the relaxation times of the labile component, while short relaxation times of crystalline water are poorly resolved. Hence, the ratio of crystalline to labile water should be taken from the solidecho measurements. The spin-echo data revealed that, upon drying, the relaxation time of the labile component significantly shortens from $193 \mu \mathrm{s}$ to $80 \mu \mathrm{s}$, which is accompanied by a 
significant reduction of its contribution to the overall signal intensity. Overall, the NMR analysis indicates that the water molecules are bound either as crystalline water $(\sim 74 \%)$ or as more loosely attached surface water, bound by weak hydrogen bonds and van der Waals interactions ( $26 \%)$. When exposed to dry atmosphere, the loosely attached water molecules are selectively desorbed, leaving the crystalline water and more tightly bound labile components behind.

These results are in good agreement with the gravimetric desorption study, since the drying process of the sample was conducted at room temperature for the NMR data. The $30{ }^{\circ} \mathrm{C}$ desorption trace recorded a weight loss of $3.5 \%$, while at slightly higher temperatures $\left(\geq 40^{\circ} \mathrm{C}\right)$ the weight loss saturates at $7 \%$, indicating the removal of close to the entire labile water fraction. This observation, together with the determined stoichiometry of $\mathrm{MoS}_{3}{ }^{2} / 3$, leads to the conclusion that 0.9 molecule of $\mathrm{H}_{2} \mathrm{O}$ is reversibly adsorbed for each Mo center. Considering the NMR results of the hydrated sample an additional 2.5 molecules of crystalline water are estimated to be bound per Mo unit, leading to a stoichiometry of $\mathrm{MoS}_{3}{ }^{2} /$. $3.4 \mathrm{H}_{2} \mathrm{O}$.

Moisture-Dependent Structural and Electronic Properties. Fourier transform infrared spectroscopy (FTIR) of dried and ambient atmosphere exposed samples revealed minor changes associated with the water bands at approximately 1580 and $3300 \mathrm{~cm}^{-1}$ upon drying (see Figure SI-5). Raman spectroscopy is better suited to investigate modulations of the Mo-S and S-S modes since their IR-active modes are only observable in the region below $500 \mathrm{~cm}^{-1}$, which is difficult to resolve using standard FTIR equipment. ${ }^{55}$ As a result, Raman spectroscopy was conducted in order to determine the nature of the water-binding sites (Figure $4 \mathrm{a}$ ). The Mo-S vibration modes are located in between 250 and $400 \mathrm{~cm}^{-1}$. The mode associated with the apical sulfur atoms is located at $445 \mathrm{~cm}^{-1}$, while $\mathrm{S}-\mathrm{S}$ modes of the terminal and bridging/shared disulfide ligands are observed at 515 and $550 \mathrm{~cm}^{-1}$, respectively. Interestingly a pronounced $\mathrm{Mo}-\mathrm{O}$ peak is seen at $940 \mathrm{~cm}^{-1}$, confirming that a significant number of oxygen defects are present in the synthesized material. ${ }^{11}$

When comparing the spectra of the dried sample with the one exposed to ambient atmosphere, it can be found that both spectra are remarkably similar to the distinction that the mode associated with the bridging and shared disulfide ligands is significantly enhanced upon exposure to atmospheric moisture. This constitutes strong evidence that the labile water molecules are bound in close proximity to the shared/bridging disulfide ligands. Recent investigations into the hydrogen-binding energy of various sites on a $\mathrm{Mo}_{3} \mathrm{~S}_{13}{ }^{-2}$-based polymer chain by Ting et al. revealed that both the bridging and terminal disulfide ligands have a high affinity toward hydrogen. ${ }^{12}$ They concluded that the binding strength at the terminal sites is large enough to form irreversible binding, while adsorption at the bridging disulfides is likely reversible, which would allow the formation of hydrogen bonds. These assertions are supported by experimental evidence reported by Afanasiev et al. ${ }^{13}$ We furthermore observed a shift of the Raman mode associated with the apical sulfide. This observation has been linked to slight distortions of the $\mathrm{Mo}_{3} \mathrm{~S}_{13}$ cluster. ${ }^{11}$

From such observations, we postulate that the bridging disulfides of the $\mathrm{MoS}_{3}{ }^{2}{ }_{3}$ polymer interact with water molecules in ambient air to engage in hydrogen bonding, leading to the reversible adsorption of the water molecules onto the $\mathrm{MoS}_{3}{ }^{2} / 3$
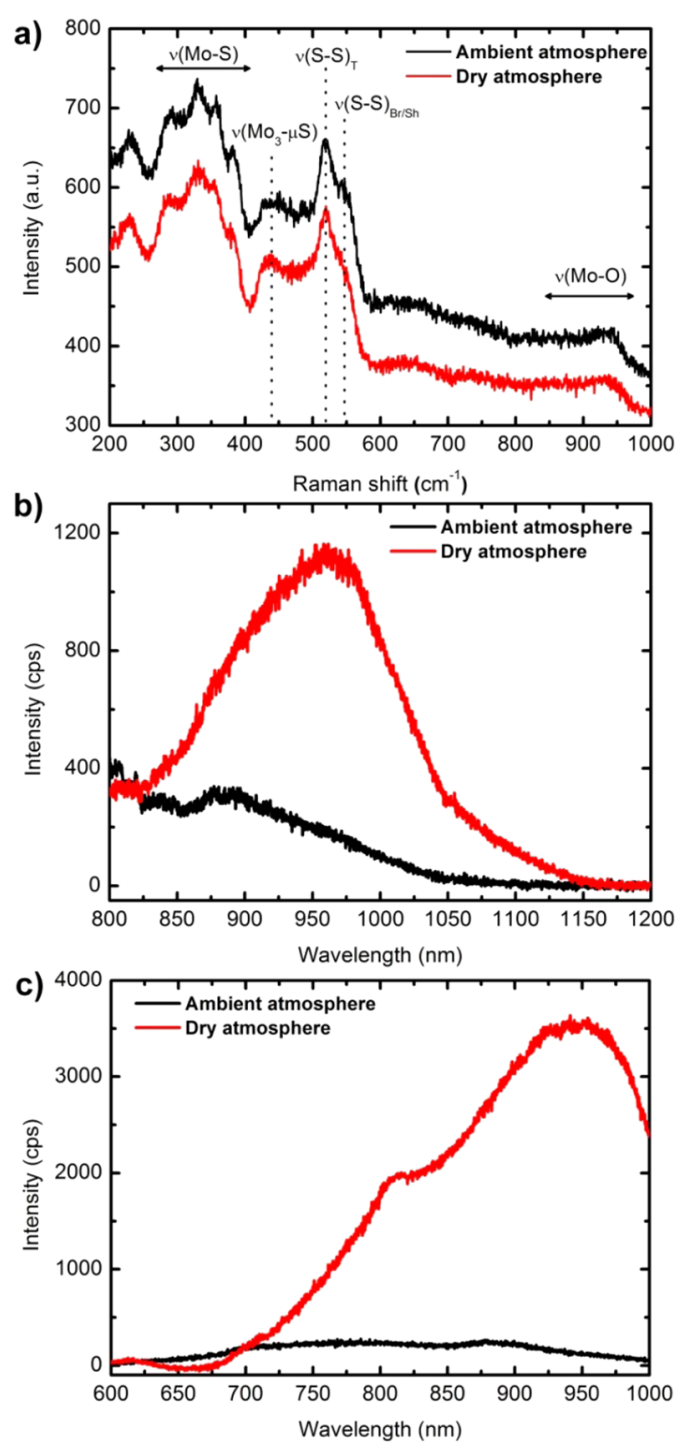

Figure 4. Raman and PL spectra of the hydrated and dehydrated $\mathrm{MoS}_{3^{2} / 3}$ samples: (a) Raman spectra recorded with an excitation wavelength of $532 \mathrm{~nm}$ on samples placed in a sealed chamber. The sample was first dried by purging the chamber with nitrogen for 4 h. The sample was then exposed to ambient atmospheric conditions, leading to the adsorption of moisture. (b and c) PL spectra of dried and moisture-exposed $\mathrm{MoS}_{3}{ }^{2} / 3$ samples excited at (b) $785 \mathrm{~nm}$ and (c) $532 \mathrm{~nm}$. For these experiment, a drop-casted sample of $\mathrm{MoS}_{3}{ }^{2} / 3$ was placed inside a sealed chamber equipped with micromanipulators, a quartz window, and a gas flow-through system. The sample was purged with nitrogen at room temperature to remove the labile water component. A Raman and PL spectrum was collected while the sample remained under a nitrogen atmosphere. Afterward the sample was exposed to ambient atmosphere and another Raman and PL spectrum was collected. The Raman spectrum of the sample exposed to air is in excellent agreement with previous reports. ${ }^{11}$

surface at the bridging and shared disulfide sites. XRD data (Figure SI-3) revealed minor shifts of the amorphous features upon drying, which indicates that the surface-adsorbed water molecules induce a slight deformation of the $\mathrm{MoS}_{3}{ }^{2} /{ }_{3}$ polymer chains, which is consistent with the occurrence of van der Waals and hydrogen bond interactions. 
We furthermore conducted photoluminescence (PL) spectroscopy inside the sealed chamber on dried and moistureexposed samples, akin to the Raman measurements (Figure $4 \mathrm{~b}$ and c). It is observed that the PL intensity of the dried $\mathrm{MoS}_{3}{ }^{2} / 3$ film is significantly higher when compared to the ambient air exposed sample. Two broad PL peaks are observed at 810 and $940 \mathrm{~nm}$ for the dried sample. Upon exposure to moisture, both PL peaks experienced a hypsochromic shift to 775 and $883 \mathrm{~nm}$. The existence of PL in the sample indicates that the material exhibits semiconducting behavior, which is in accordance with previous reports. ${ }^{28,56}$ The quenching of the PL, together with the shift of the luminescence, indicates that exposure to moisture significantly alters the electronic properties of the material. The quenching is particularly revealing, indicating that significant charge transfer from the water molecules to the $\mathrm{MoS}_{3}{ }^{2} / 3$ chain occurred, which supports the hypothesis that hydrogen bonds are formed.

Relative Humidity Sensing. The observed electronic changes and fast moisture adsorption and desorption kinetics indicate that the materials can be exploited for moisture sensing. For this purpose, a thin film of $\mathrm{MoS}_{3}{ }^{2} / 3$ was drop casted onto interdigitated electrodes (Figure 5a) to create a conductometric moisture sensor. The sensor's resistance was then measured while being exposed to gas flows with controlled

a)

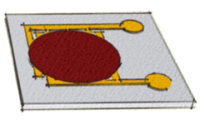

$\mathrm{H}_{2} \mathrm{O}$ vapor
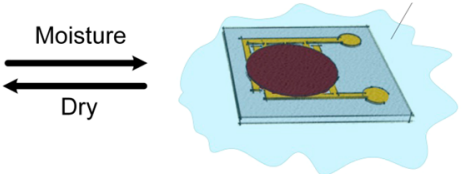

b)

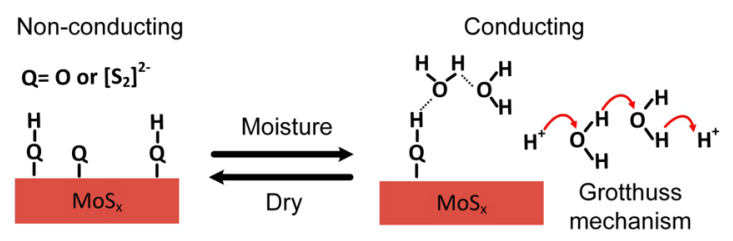

c)

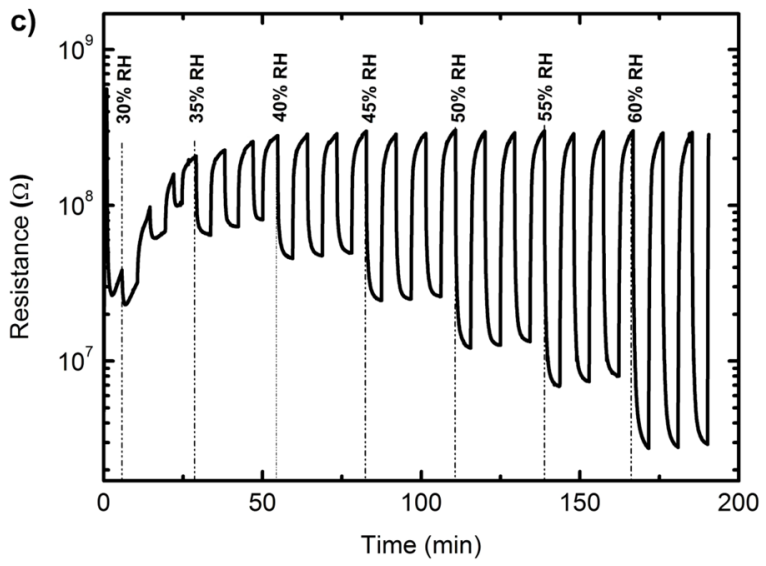

Figure 5. Demonstration of the moisture-sensing concept: (a) schematic representation of the device, (b) suggested sensing mechanism based on physisorbed moisture and proton conduction based on the Grotthuss mechanism, (c) measured resistance of the sensor when exposed to defined gas flows with specified relative humidity. Three cycles were measured for each gas flow. In between each cycle the sensor was exposed to a baseline of $25 \% \mathrm{RH}$ (at $30^{\circ} \mathrm{C}$ ). relative humidity $(\mathrm{RH})$. Upon exposure to dry atmosphere, the resistance of the sensor rapidly increased by over 3 orders of magnitude, beyond the upper detection limit of our characterization equipment. This finding confirmed that $\mathrm{MoS}_{3}{ }^{2} / 3$ transitioned from a conductive state to being an insulator due to the desorption of water.

We then investigated the sensor's dynamic response to humidity changes between $30 \%$ and $60 \% \mathrm{RH}$ with a baseline of $25 \% \mathrm{RH}$ (Figure 5c). The baseline of $25 \% \mathrm{RH}$ was selected since atmospheres with lower moisture content resulted in resistance values above the detection limit of our equipment. This limitation may be overcome in the future by optimizing the spacing in between the interdigitated electrodes. The developed sensor showed a logarithmic dependency of the resistance on the relative humidity of the gas (Figure SI-6).

In order to determine possible sensing mechanisms, the nature of the majority charge carriers was determined using electrochemical Mott-Schottky analysis (Figure SI-7). ${ }^{59}$ $\mathrm{MoS}_{3}{ }^{2} / 3$ was found to be a p-type semiconductor, indicating that the samples' conductivity is not dominated by its semiconducting properties in response to humidity. Conductivity of p-type semiconductors is well known to decrease upon exposure to moisture due to the observed modulation of their electronic structure. ${ }^{44}$ The previously discussed PL characterization revealed that the exposure to moisture results in the modulation of the electronic properties of $\mathrm{MoS}_{3}{ }^{2} / 3$, which indicates that the presence of surface water alters the density of states within the semiconductor. The modulation of the density of states of the semiconductor may not necessarily involve a complete charge transfer but can be induced via dipole interactions and small distortions of the molecular structure. ${ }^{58}$ The presence of structural distortions upon moisture adsorption has also been implicated during our Raman spectroscopy and XRD analysis. However, photoconductivity measurements revealed a negligible change in resistance when illuminating the sensor in ambient air (Figure SI-8). Additionally, the resistivity decreases in response to an increase in humidity. These measurements lead to the conclusion that a charge injection based mechanism is occurring but will not be the dominant cause of the humidity sensor response.

A more likely explanation for the observed moisturedependent conductivity modulation is based on well-known proton conductivity within the physisorbed surface water. ${ }^{44}$ As discussed within the context of the low-field NMR analysis, a large amount of moisture may be bound to the surface of $\mathrm{MoS}_{3{ }^{2} / 3}$ via hydrogen bonding. Possible binding sites are surface-bound hydrogen atoms, which in this case are likely present in the form of surface hydroxyl and protonated disulfide groups (Figure 5b). ${ }^{11,12}$ In a high-humidity environment, multiple layers of hydrogen-bonded water molecules may accumulate, allowing efficient proton conduction based on the Grotthuss mechanism, ${ }^{44,59}$ accounting for the significant decrease in resistivity upon exposure to moisture (see Figure 5). Proton conductivity based sensing mechanisms are known to frequently dominate the response of high surface area moisture-sensing materials at room temperature. ${ }^{44}$

The effect of the conductivity change of the semiconductor is likely to be much smaller in comparison to the variations in dominant proton conductivity on the very large surface area of the synthesized material. As such, the overall resistivity change in response to humidity increase is a large reduction in magnitude. 
Overall, we observed a change in resistivity of over 2 orders of magnitude between $25 \%$ and $60 \% \mathrm{RH}$ (at $30{ }^{\circ} \mathrm{C}$ ). The sensor's device parameters measured for various water concentrations are presented in Table 1 . The response times

Table 1. Measured Sensor Characteristics of the Developed $\mathrm{MoS}_{3}{ }^{2} / 3$-Based Humidity Sensor ${ }^{a}$

$\begin{array}{cccc}\mathrm{RH}\left(30^{\circ} \mathrm{C}\right)(\%) & t_{\text {res }}(\mathrm{s}) & t_{\text {rec }}(\mathrm{s}) & \left|R_{0} / \Delta R\right| \\ 35 & 58 & 167 & 4.0 \\ 40 & 31 & 203 & 6.2 \\ 45 & 28 & 205 & 11.8 \\ 50 & 20 & 196 & 23.3 \\ 55 & 23 & 211 & 39.8 \\ 60 & 16 & 203 & 104.8\end{array}$

$a_{\text {The response time }} t_{\text {res }}$ and recovery time $t_{\text {rec }}$ are defined as the time necessary to reach $90 \%$ of the total signal height and recovery to $90 \%$ of the baseline; the sensitivity $\left(\left|R_{0} / \Delta R\right|\right)$ is defined as the ratio between the baseline resistance $R_{0}$ at equilibrium and the total change of the resistance $(\Delta R)$. The base load was $25 \% \mathrm{RH}$ (at $30^{\circ} \mathrm{C}$ ). Values are given for the second of three consecutive measurements.

of the senor were determined to be between 58 and $16 \mathrm{~s}$, with a faster response associated with a larger change in relative humidity. The recovery time was found to be around $200 \mathrm{~s}$. The sensitivity of the sensor $\left(\left|R_{0} / \Delta R\right|\right)$ is defined as the baseline value $\left(R_{0}\right)$ relative to the total change of the device's resistance $(\Delta R)$ and was found to be between 4.0 and 104.8 for relative humidities of $35 \%$ and $60 \%$ (at $30{ }^{\circ} \mathrm{C}$ ), respectively, indicating that the resistance dropped by $75 \%$ to $99 \%$. Interestingly the sensor showed no response to $\mathrm{NO}_{2}$ or $\mathrm{H}_{2} \mathrm{~S}$ gas (data not shown), highlighting good selectivity. Successive measurements indicate that the device is very stable and features minimal hysteresis.

Altogether the humidity sensor's performance is well suited for practical applications and the performance parameters are on par with state-of-the-art materials. ${ }^{43,60}$ Semiconducting moisture sensors including those that rely on proton conduction are typically oxides, due to the crucial role of the surface oxide sites in the established sensing mechanism. ${ }^{44}$ In this work, the previously identified surface hydroxyl and protonated disulfide groups may act as initial binding sites, followed by further physisorption utilizing hydrogen bonds. ${ }^{44}$

For comparison moisture sensors were fabricated using bulk $\mathrm{MoS}_{2}$ and PAAHCl. Samples containing bulk $\mathrm{MoS}_{2}$ and a mixture of $\mathrm{MoS}_{2}$ and $\mathrm{PAAHCl}$ were prepared following the same vapor phase acidification and subsequent workup procedure that was employed to prepare $\mathrm{MoS}_{3}{ }^{2} / 3$. One further sample was prepared using an aqueous solution of PAAHCl. The $\mathrm{MoS}_{2}$-containing samples showed nearly 4 orders of magnitude smaller response to moisture, highlighting the exceptional performance of $\mathrm{MoS}_{3}{ }^{2} / 3$ (see Figure SI-9). The PAAHCl-based sensor was fabricated to ensure that the observed response did not originate from template residues that may be present at concentrations below the detection limit of Raman spectroscopy. The PAAHCl-based sensor did not show any detectible response to moisture exposure, and thus it is concluded that the measured conductivity change of the $\mathrm{MoS}_{3 / 3}{ }^{2}$ sample is indeed associated with $\mathrm{MoS}_{3}{ }^{2} / 3$.

Electrolyteless Water Splitting. As discussed previously, one of the key applications of $\mathrm{MoS}_{3}{ }^{2} / 3$ can potentially be in the field of hydrogen production, where it commonly serves as an electrocatalyst. Considering the observed rapid moisture capture and good conductivity of the synthesized material, we explored its application toward electrolyte-free hydrogen production. For this purpose, we formulated a catalytic ink that can be utilized for $\mathrm{H}_{2}$ production without the need of any external power sources or fluid handling equipment. The development of an ink-based electrolyteless gas phase photocatalytic hydrogen evolution catalyst is highly attractive, since it significantly simplifies the device structure, essentially allowing to adapt any surface toward solar fuel production. Since the catalytic process is electrolyte free, the fuel production is not only carbon neutral but also water neutral, not relying on drinking water to sustain its operation. ${ }^{5}$ Furthermore, the aforementioned disadvantages of using electrolytes such as the risk of freezing, leakage, and corrosion are entirely avoided. ${ }^{5}$

Since the band gap of $\mathrm{MoS}_{3}{ }^{2} / 3$ is too narrow to facilitate water splitting on its own, a mixture of $\mathrm{MoS}_{3}{ }^{2} / 3$ and $\mathrm{TiO}_{2}$ (P25) was designed. Amorphous molybdenum sulfides are frequently utilized in conjunction with wide band gap semiconductors such as $\mathrm{CdS}$ and $\mathrm{TiO}_{2}$ or organic sensitizers such as erythrosine B. ${ }^{31,35}$ The wide band gap material promotes light harvesting and provides high-energy charge carriers to the catalytically active molybdenum sulfide, leading to hydrogen and oxygen evolution (Figure 6b). In the envisaged design, sulfur-rich $\mathrm{MoS}_{3{ }^{2} / 3}$ captures water from moist air and then acts as a photocatalyst in conjunction with $\mathrm{TiO}_{2}$. Figure $6 \mathrm{a}$ and b show a schematic representation of the catalytic device and an energy diagram highlighting the active components.

The conduction band positions of $\mathrm{MoS}_{3}{ }^{2} /_{3}$ and $\mathrm{TiO}_{2}$ were determined using Mott-Schottky analysis (Figure SI-7) in buffered $\mathrm{KCl}$ solutions with close to neutral $\mathrm{pH}$ ( $\mathrm{pH}$ 8.1). For the $\mathrm{MoS}_{3}{ }^{2} / 3$ sample the valence band position is determined, which in conjunction with the UV-vis spectroscopy results (Figure 2) provides the position of the conduction band. ${ }^{57}$ Mott-Schottky plots of n-type semiconductors such as $\mathrm{TiO}_{2}$ directly indicate the position of the conduction band. ${ }^{61}$ The conduction band edge of $\mathrm{MoS}_{3}{ }^{2} / 3$ is found to be well positioned to facilitate $\mathrm{H}_{2}$ evolution, as well as to be sensitized by $\mathrm{TiO}_{2}$. Oxygen evolution is expected to be facilitated by $\mathrm{TiO}_{2}$, since the valence band of $\mathrm{MoS}_{3}{ }^{2} / 3$ is not likely to be oxidizing enough to directly evolve oxygen. ${ }^{62}$ In order to confirm that the band positions are not altered upon blending $\mathrm{MoS}_{3}{ }^{2}{ }_{3}$ and $\mathrm{TiO}_{2}$ during the ink formulation, we proceeded to conduct photoelectron spectroscopy in air (PESA). PESA is well suited to investigate the band positions of mixed systems containing wide band gap semiconductors as well as narrow band gap components. ${ }^{63}$ PESA measurements of only $\mathrm{MoS}_{3{ }^{2} / 3}$ and the mixed $\mathrm{MoS}_{3{ }^{2} / 3} / \mathrm{TiO}_{2}$ system are shown in Figure SI-10. Due to the very low position of the $\mathrm{TiO}_{2}$ valence band, only photoelectron emission from $\mathrm{MoS}_{3}{ }^{2} / 3$ is observed. The measured data confirmed that blending the individual components did not alter the band positions of $\mathrm{MoS}_{3}{ }^{2} / 3^{\prime}$ supporting the validity of the developed energy diagram displayed in Figure $6 \mathrm{~b}$. Variations between the absolute band position observed using PESA and Mott-Schottky analysis can be attributed to differences of the $\mathrm{pH}$ in the surface-absorbed moisture layer (PESA measurement) and the buffered solution (Mott-Schottky analysis). 

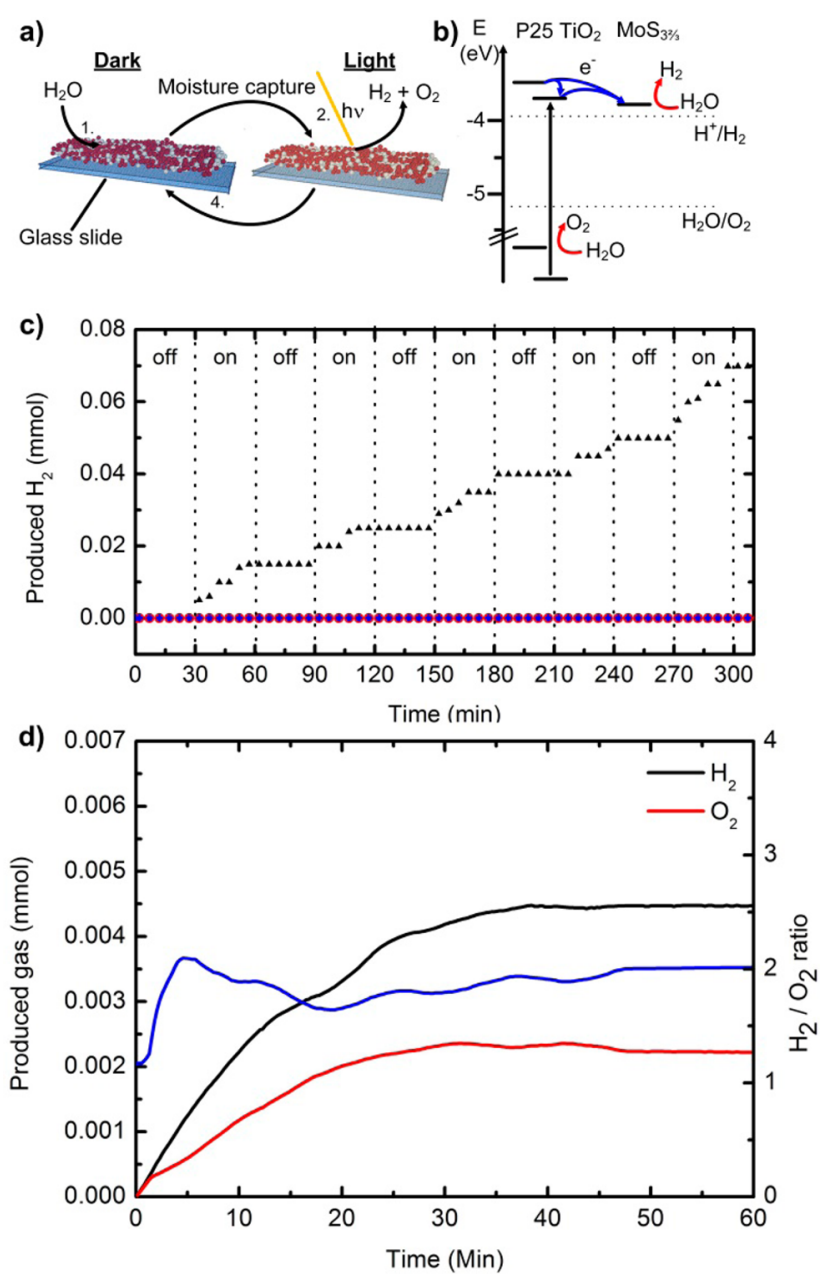

Figure 6. Demonstration of electrolyteless gas phase water splitting: (a) schematic of the catalytic device and the cyclic operation. Step 1 indicates the initial water adsorption by $\mathrm{MoS}_{3}{ }^{2} / 3$ prior to catalysis. Once illuminated, the $\mathrm{TiO}_{2}$ and $\mathrm{MoS}_{3}{ }^{2}{ }_{3}$ particles harvest light (step 2), which leads to overall water splitting (step 3). The device recaptures the lost water molecules during the dark cycle (step 4). (b) Energy diagram of the catalyst. The conduction band position of $\mathrm{P} 25 \mathrm{TiO}_{2}$ was measured using Mott-Schottky analysis, and the respective relative values for the anatase and rutile components were taken from the literature. ${ }^{69}$ The conduction band position of $\mathrm{MoS}_{3}{ }^{2} / 3$ was determined using a combination of $\mathrm{UV}-$ vis spectroscopy and Mott-Schottky analysis. All values are given for an aqueous environment of $\mathrm{pH}$ 8.1. (c) Measured evolved hydrogen during cyclic catalysis using $\mathrm{TiO}_{2}$ (blue), $\mathrm{MoS}_{3}{ }^{2} / 3$ (red), and a mixture of $90 \% \mathrm{MoS}_{3}{ }^{2} / 3$ and $10 \% \mathrm{TiO}_{2}$ by weight (black). (d) Photocatalytic hydrogen (black) and oxygen (red) production of a printed photocatalyst layer containing $90 \% \mathrm{MoS}_{3}{ }^{2} / 3$ and $10 \% \mathrm{TiO}_{2}$ (P25), measured under an anaerobic $\left(\mathrm{N}_{2}\right)$ condition. The blue line indicates the hydrogen to oxygen ratio.

The optimized ink formulation consisted of a simple $90 \%$ $\mathrm{MoS}_{3}{ }^{2} / 3$ and $10 \% \mathrm{TiO}_{2}$ containing aqueous suspension with a combined solid loading of $20 \%$, which could be deposited onto a glass substrate using the doctor blade technique. The sample was then placed in a sealed glass vessel with a moisturesaturated atmosphere. The vessel was equipped with a custombuilt hydrogen headspace sensor, which recorded the hydrogen concentration during illumination with simulated solar light.
Hydrogen production was detected when the $\mathrm{MoS}_{3}{ }^{2}{ }_{3} / \mathrm{TiO}_{2}$ mixture was illuminated, while films containing only $\mathrm{MoS}_{3{ }^{2} / 3}$ or $\mathrm{TiO}_{2}$ resulted in no detectable $\mathrm{H}_{2}$ concentration (Figure $6 \mathrm{c}$ ), indicating that the formation of a heterojunction is paramount to the process. To ensure that overall water splitting is achieved, oxygen and hydrogen evolution measurements were conducted (see Figure 6d). The ratio of $\mathrm{H}_{2}$ to $\mathrm{O}_{2}$ was found to be $2: 1$, indicating that stoichiometric overall water splitting is achieved. We observed, however, that the $\mathrm{H}_{2}$ production rate of the optimized ink declined upon prolonged illumination (Figure 6d and SI-11) and eventually ceased. To our pleasant surprise, the film regained its catalytic activity upon storage in the dark, which allowed us to design a cyclic illumination pattern, which enables continued hydrogen production. Figure $6 \mathrm{c}$ shows the cumulative hydrogen production observed after 5 cycles of $30 \mathrm{~min}$ illumination followed by $30 \mathrm{~min}$ of storage in a dark humid environment. We postulate that the catalytic system loses its moisture content under illumination due to the desired catalytic water splitting and thermal effects, to a point where sustained $\mathrm{H}_{2}$ production is not possible. The recapture rate of $\mathrm{H}_{2} \mathrm{O}$ by the catalyst, while under illumination, appears to be too slow to sustain continuous water splitting under one sun illumination. The relative humidity of the atmosphere within the reaction chamber was measured to be $93 \%$, indicating that simply increasing the humidity of the system is not likely to overcome this limitation. Measurements of the $\mathrm{MoS}_{3}{ }^{2} / 3 / \mathrm{TiO}_{2}$ film temperature within the reaction chamber revealed that the temperature of the catalyst layer rapidly increases upon illumination, reaching an equilibrium operating temperature of $60{ }^{\circ} \mathrm{C}$ within 8 to $10 \mathrm{~min}$ (see Figure SI-12). Considering the previously discussed thermal desorption measurements, which revealed rapid moisture loss at $40-60{ }^{\circ} \mathrm{C}$, thermally induced moisture loss is the most likely cause for the temporary decline in the $\mathrm{H}_{2}$ production and the need for cyclic illumination.

Light absorbed by $\mathrm{MoS}_{3}{ }^{2} / 3$ is expected to not fully contribute to the overall water splitting reaction, due to the narrow band gap. The valence band of $\mathrm{MoS}_{3}{ }^{2} / 3$ is not likely to be oxidizing enough to directly evolve oxygen, while the conduction band is well positioned to facilitate hydrogen evolution. Trapping of the photogenerated holes in $\mathrm{MoS}_{3}{ }^{2} / 3$ is avoided due to the low diffusion length of holes in $\mathrm{TiO}_{2}\left(\sim 10^{-9} \mathrm{~m}\right) .{ }^{64}$ Photogenerated electrons with sufficient reduction potential to facilitate hydrogen evolution, on the other hand, are directly produced in $\mathrm{MoS}_{3}{ }^{2} / 3$ and can also be transferred to $\mathrm{MoS}_{3}{ }^{2} / 3$ from $\mathrm{TiO}_{2}$ due to the comparatively large diffusion length of electrons in $\mathrm{TiO}_{2}\left(\sim 10^{-5} \mathrm{~m}\right) .^{65}$ As such, the developed energy diagram and operating principle are comparable to noble metal functionalized wide band gap photocatalysts with the advantageous distinction that the hygroscopic nature of $\mathrm{MoS}_{3}{ }^{2} / 3$ facilitates electrolyteless operation. ${ }^{66}$ Oxygen evolution is expected to be the limiting step for overall water splitting, due to the wide band gap of $\mathrm{TiO}_{2}$, resulting in thermal heating due to this mismatch. Hence during cyclic operation, the adsorbed water is consumed under illumination to produce hydrogen, while the hygroscopic $\mathrm{MoS}_{3{ }^{2} / 3}$ chains recapture thermally desorbed and decomposed water during the dark resting phase (Figure 6a).

The hydrogen production rates were found to be 11.09 and $4.03 \mathrm{mmol} \mathrm{g}^{-1} \mathrm{~h}^{-1}$ for the cyclic illumination and continuous illumination strategies, respectively. The hydrogen production 
rates translate to 2.48 and $0.90 \mathrm{mmol} \mathrm{m} \mathrm{m}^{-2} \mathrm{~h}^{-1}$, respectively, when the Brunauer-Emmett-Teller (BET) surface area (4.48 $\mathrm{m}^{2} \mathrm{~g}^{-1}$ ) of the photocatalyst is considered. It is noteworthy to highlight that the BET surface area measured using inert gases likely underestimates the available surface area for $\mathrm{H}_{2} \mathrm{O}$ adsorption. The average turnover frequencies for a $1 \mathrm{~h}$ reaction period were calculated to be 940 and $342 \mu \mathrm{Hz}$ for cyclic and continuous illumination patterns, respectively. ${ }^{67}$ The demonstrated electrolyteless hydrogen evolution system is surprisingly efficient considering that it relies entirely on the capture of moisture from humid air. The developed system outperforms previous reports on gas phase electrolyteless photocatalytic water splitting (see Table 2). Considering that previous works used high-intensity UV illumination rather than simulated solar light indicates orders of magnitude improved $\mathrm{H}_{2}$ production rates under real-world application conditions. For comparison,

Table 2. Comparison of Different Gas Phase Hydrogen Evolution Water Splitting Systems ${ }^{a}$

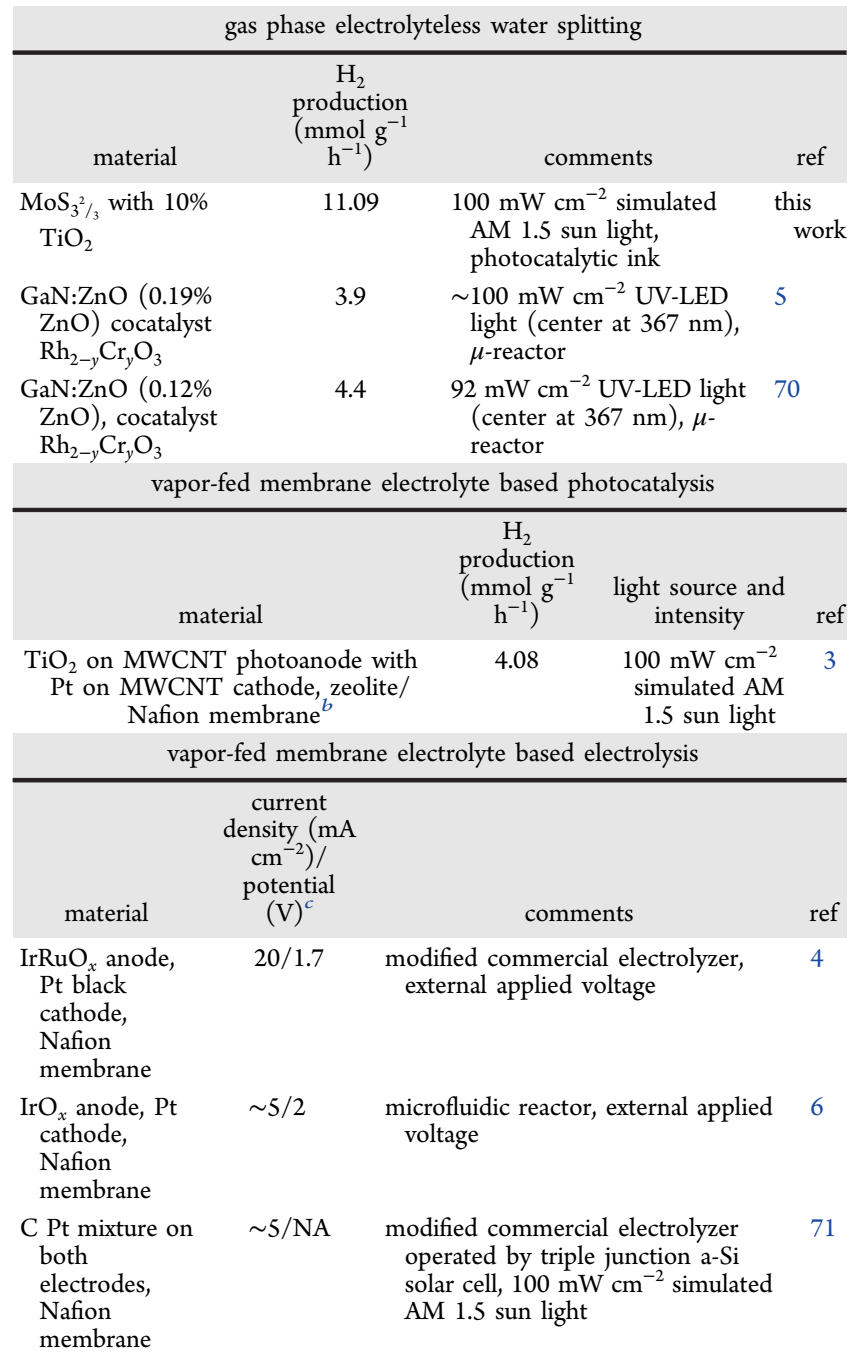

${ }^{a}$ Note: when comparing different catalysts' performance, the illumination source needs to be considered. Many reported $\mathrm{H}_{2}$ production rates were conducted using light sources that emit strongly in the UV region, leading to much higher $\mathrm{H}_{2}$ production rates then what would be expected under simulated solar light. ${ }^{b} \mathrm{MWCNT}$ abbreviates multiwalled carbon nanotubes. ${ }^{c} 1 \mathrm{~mA} \mathrm{~cm}^{-2}$ converts to a hydrogen production rate of $0.0187 \mathrm{mmol} \mathrm{cm} \mathrm{ch}^{-2} \mathrm{~h}^{-1}$ vapor fed membrane electrolyte based photocatalytic and electrolysis systems are also included in Table 2. The $\mathrm{H}_{2}$ production rates can likely be improved further by employing semiconductor nanoparticles with a more favorable absorption profile than $\mathrm{TiO}_{2}$ as cocatalyst and by optimizing the design of the device, for example by utilizing a transparent $3 \mathrm{D}$ support that allows higher catalyst loading and optimizes light absorption and gas transport. ${ }^{62,68}$ This, in conjunction with improved thermal management, may also allow overcoming the need for a cyclic illumination pattern.

\section{CONCLUSIONS}

We demonstrated the synthesis of highly porous sulfur-rich $\mathrm{MoS}_{3{ }^{2} / 3}$ utilizing a facile and scalable acidification-polymerization route, employing a cationic templating agent. We proceeded to characterize the interactions between this inorganic polymer and moist air. It was discovered that $\mathrm{MoS}_{3}{ }^{2} / 3$ can reversibly bind up to $7 \%$ water by weight $\left(0.9 \mathrm{H}_{2} \mathrm{O}\right.$ per Mo center) in ambient conditions, which can be released at low temperatures in dry atmospheres. This effect may be utilized to design low-energy dehumidification devices. Lowfield NMR studies revealed that the moisture is adsorbed as weakly bound surface water, likely utilizing van der Waals forces and hydrogen bonding. Raman spectroscopy studies revealed that the reversible binding of water molecules likely occurs at the shared and bridging disulfide ligands that link the individual Mo centers within the porous and branched structure.

We furthermore observed that the binding of the water molecules had a profound impact on the optical and electronic properties of $\mathrm{MoS}_{3}{ }^{2} / 3$. When stored under nitrogen, intense luminescence in the near-infrared region is observed, while a distinct hypsochromic shift and PL quenching occur upon exposure to atmospheric moisture. The observed quenching of PL was attributed to charge transfer processes due to van der Waals interactions and hydrogen bonding. The material was found to be insulating when exposed to dry conditions, while the adsorption of surface water resulted in markedly reduced impedance, which was attributed to proton conduction. This observation was utilized to design moisture sensors, featuring high sensitivity and selectivity to $\mathrm{H}_{2} \mathrm{O}$ at room temperature.

While these observations are highly relevant on their own, they also have significant implications to the field of hydrogen evolution catalysis, which is one of the most prominent applications of amorphous molybdenum sulfides. The observed affinity of the bridging and shared disulfide ligands toward water molecules implicates their significance to the overall water splitting process. Furthermore, the observed dependence of the electronic and optical properties on the amount of surface-adsorbed water molecules should be considered when characterizing this material in the future.

Finally we developed a photocatalytically active ink based on sulfur-rich $\mathrm{MoS}_{3}{ }^{2} / 3$ combined with $\mathrm{TiO}_{2}$ nanoparticles, which function as a photosensitizer and cocatalyst. Printed films of this composite were found to be catalytically active in moist atmospheres, without the need for any electrolytes or external power sources. While the overall hydrogen production efficiency is lower than what is typically observed when using catalysts suspended in electrolytes, the process is surprisingly efficient considering that it relies entirely on the here discovered hygroscopic nature of $\mathrm{MoS}_{3}{ }^{2} / 3$ as a water source. A hydrogen evolution rate of $11.09 \mathrm{mmol} \mathrm{g}^{-1} \mathrm{~h}^{-1}$ could be 
achieved under optimized conditions, which also resulted in a much higher efficiency than any previously reported photocatalytic gas phase water splitting system. Pending future improvements of the process efficiency, electrolyteless photocatalytic water splitting may become an attractive alternative approach due to its unique features and simplicity, and hence our findings are expected to stimulate further work within the field.

\section{METHODS}

Synthesis of Partially Hydrated $\mathrm{MoS}_{3}{ }^{2} /_{3}$. A facile templated acidification polymerization method was used for synthesizing hydrated $\mathrm{MoS}_{3}{ }^{2} / 3$ suspensions. A $\left(\mathrm{NH}_{4}\right)_{2} \mathrm{MoS}_{4}$ precursor was employed in conjunction with the cationic polymer PAAHCl. All reagents were purchased from Sigma-Aldrich and used directly without any additional purification. In a typical synthesis process, $260.3 \mathrm{mg}$ of $\left(\mathrm{NH}_{4}\right)_{2} \mathrm{MoS}_{4}$ and $187.1 \mathrm{mg}$ of PAAHCl were dissolved in $10 \mathrm{~mL}$ of deionized (DI) water in a glass vial. The color of the solution was found to rapidly transition from red to turbid orange due to the binding of the tetrathiomolybdate ions to the cationic templates. The solution was then acidified by placing $2-3 \mathrm{~mL}$ of concentrated $\mathrm{HCl}$ $(32 \%)$ next to the sample in a closed system for $24 \mathrm{~h}$. The presence of $\mathrm{HCl}$ vapors led to a gradual acidification of the sample, and the color of the solution changed from bright orange to black overnight. The solution was stirred throughout the process. The product was then isolated by centrifugation at $2500 \mathrm{rcf}$ for $1 \mathrm{~h}$. In order to remove any residual educts, the templating polymer, $\mathrm{HCl}$, and side products, the product was redispersed into $10 \mathrm{~mL}$ of DI water and re-collected by centrifugation. This washing step was repeated twice to ensure the successful removal of all contaminations. Raman spectroscopy was utilized to ensure that the templating polymer was successfully removed. A part of the sample was then dried on a large glass Petri dish at room temperature for 3 days for further analysis that required solid samples.

Fabrication of Moisture Sensor. Approximately $100 \mu \mathrm{L}$ of the $\mathrm{MoS}_{3 / 3}{ }^{2}$ suspension was drop casted onto an interdigitated electrode on an alumina ceramic substrate with $\mathrm{Ag}-\mathrm{Pd}$ electrodes, purchased from Elite Tech (Figure SI-13 for the design). The sensor was dried at room temperature overnight prior to testing. Care was taken to ensure that the entire electrode area was covered with a thin film of the sample. For comparison samples of bulk $\mathrm{MoS}_{2}$ (US-nano, 99.9\%, 800-1200 nm size) and bulk $\mathrm{MoS}_{2}$ mixed with the polymer template (PAAHCl) were processed following the same sample preparation protocol that was utilized to create the $\mathrm{MoS}_{3^{2} / 3}$ sample (vapor phase acidification and reaction workup procedure). The same ratio of Mo to the polymer and overall Mo concentration were used for consistency. Similarly a solution of $187.1 \mathrm{mg}$ of PAAHCl in DI water was prepared. These three samples were employed to prepare further moisture sensors as reference samples following the method described previously.

Photocatalytic Device Fabrication. For the photocatalytic experiments an ink was formulated that could be deposited onto microscopy glass slides. The optimized ink contained $900 \mathrm{mg}$ of dried $\mathrm{MoS}_{3 / 3}{ }^{2}$ and $100 \mathrm{mg}$ of $\mathrm{TiO}_{2} \mathrm{P} 25$ (Sigma-Aldrich). The solid components were mixed with $5 \mathrm{~mL}$ of DI water and sonicated in a bath sonicator for $1 \mathrm{~h}$. The ink was then deposited onto microscopy glass slides using the doctor blade technique with Scotch tape to define the thickness of the film and the area. The glass slides were cleaned in hot concentrated sulfuric acid for $10 \mathrm{~min}$ followed by rinsing in DI water prior to the deposition, which was found to lead to improved adhesion of the deposited film. A single print of the ink was applied followed by drying of the film at $40{ }^{\circ} \mathrm{C}$ on a hot plate. The catalyst loading was determined to be $0.6 \mathrm{mg}$ per $\mathrm{cm}^{2}$ using gravimetric analysis.

Electrolyteless Gas Phase Water Splitting. A specially designed reactor was utilized for the electrolyteless gas phase photocatalytic water splitting experiments. The reactor chamber was a $500 \mathrm{~mL}$ sealed glass vessel with a detachable headspace sensor unit. The sensor unit was built in-house utilizing two SGX Sensortech VQ546 M hydrogen sensor chips and two SGX Sensortech EC410 oxygen sensors. The sensors were calibrated prior to use with hydrogen-nitrogen standard mixtures and laboratory oxygen, which were diluted further using an MKS mass flow controller system, creating a set of accurate calibration standards. All sensors were found to function according to the manufacturer's specification. The overall pressure and temperature were recorded using an ANKOM pressure transducer fitted with a temperature sensor, which was incorporated in the sensing head. The photocatalyst-coated microscopy glass slide was placed onto two stands within the reactor vessel. A $10 \mathrm{~mL}$ amount of distilled water was placed below the catalyst to ensure that the atmosphere was saturated with water vapor. The system was allowed to reach equilibrium in the dark for $30 \mathrm{~min}$, upon which the catalytic film was illuminated with simulated solar light using a $150 \mathrm{~W}$ Xe lamp solar simulator (ABET Technologies) with 1 sun $\left(100 \mathrm{~mW} \mathrm{~cm}^{-2}\right)$ light intensity. The relative humidity within the reactor vessel was measured using a hand-held humidity sensor (Extech Advantage RH520A-240). The illuminated sample area was $3.5 \mathrm{~cm}^{2}$. The samples were allowed to equilibrate within the sealed chamber in the dark for $30 \mathrm{~min}$ prior to the experiment. The samples were then either continuously illuminated or illuminated in a cyclic pattern, being illuminated for $30 \mathrm{~min}$, followed by $30 \mathrm{~min}$ of storage in the dark. The produced hydrogen was measured using the headspace sensors, and both hydrogen sensors were in good agreement with each other.

Measurements of oxygen and hydrogen, in parallel, were conducted under anaerobic conditions using the same setup as described above. The atmospheric background pressure of oxygen was found to mask the comparatively small quantity of oxygen produced during photocatalysis. This could be overcome by working in a nitrogen atmosphere. For the experiment, the reaction chamber was purged with nitrogen for $30 \mathrm{~min}$ through a septa port fitted with a customized Luer lock fitting featuring a one-way check valve, preventing gas backflow. The machined fitting was sealed against the septa port using a Viton O-ring and secured using the standard septa port screw cap. After purging the reaction chamber the system was allowed to reach equilibrium in the dark prior to illuminating the sample as described previously.

The temperature of the printed photocatalytic film under illumination was measured during a typical electrolyteless photocatalytic water splitting experiment using a hand-held infrared noncontact thermometer.

Material Characterization. UV-vis spectroscopy was carried out using a Cary 500 spectrometer (Varian) and quartz cuvettes. Raman spectroscopy and PL spectroscopy were carried out using a Labram HR evolution spectrometer (Horiba). For this experiment the sample was drop casted onto a gold-coated silicon wafer and placed in a sealed chamber equipped with a quartz window, gas in- and outlets, and micromanipulator electrical probes (Linkam HFS600 PB4). For the Raman measurements the sample was excited with $4.5 \mathrm{~mW}, 532 \mathrm{~nm}$ light. The measurements were conducted using a $10 \times$ lens and 1800 lines per $\mathrm{mm}$ grating. The sample was purged with dry nitrogen $(200$ $\mathrm{sccm}$ ) for $4 \mathrm{~h}$ prior to the dry measurements. The sample was then exposed to ambient air $(30 \mathrm{~min})$ for the moist measurements. The PL measurements were conducted using the same instrument as for the Raman characterization, using $2.25 \mathrm{~mW}, 532$ and $785 \mathrm{~nm}$ excitation.

XPS was carried out on a Thermo K-Alpha instrument (Thermo Scientific) with monochromatic $\mathrm{Al} \mathrm{K} \alpha$ radiation $(\lambda=1486.7 \mathrm{eV})$. The samples were drop casted onto $\mathrm{Si}$ wafers for this characterization. Transmission electron microscopy was carried out on JEOL 1010 and $20100 \mathrm{~F}$ instruments operating with an 100 and $200 \mathrm{kV}$ acceleration voltage, respectively, and equipped with Gatan Orius SC600 (JEOL 1010 ) and SC1000 (JEOL 20100F) CCD cameras. The samples were drop cast onto holey carbon grids (Proscitec) for the analysis.

Low-field NMR analysis was carried out on a Bruker minispec mq20 NMR analyzer. The dried sample was stored for $100 \mathrm{~h}$ in a nitrogen glovebox prior to being sealed in the NMR tube. The measurements were carried out directly after removal from the glovebox to minimize any moisture diffusing into the sealed tube. The solid-echo and spin- 
echo pulse sequences (modified fid mb sequences) were acquired using 128 scans and a relaxation delay of $2 \mathrm{~s}$. Attenuated total reflection (ATR) Fourier transform infrared spectroscopy was conducted using a PerkinElmer Spectrum 100 spectrometer equipped with a universal ATR accessory. The samples were prepared following the same protocol as for the low-field NMR measurements. The moisture desorption isotherms were measured using a PerkinElmer Pyris 1 thermogravimetric analyzer (TGA). For this experiment approximately $4 \mathrm{mg}$ of the room-temperature dried sample was weighed into a platinum TGA pan. Once the instrument reached the desired temperature, the sample was loaded into the TGA, the sample was exposed to dry nitrogen flows with a flow rate of $20 \mathrm{sccm}$, and the sample weight was recorded. The sample was exposed to ambient air for $5 \mathrm{~min}$ between each cycle during the cyclic desorption experiments. BET surface area measurements were conducted using approximately $500 \mathrm{mg}$ of $\mathrm{MoS}_{3^{2} / 3}, \mathrm{~N}_{2}$ as the process gas, and a Micromeritic ASAP 2400 surface area analyzer.

Moisture sensing was conducted in the same sealed chamber that was used for the Raman measurements. The sensor was placed inside the chamber and connections to the contact pads were made with the micromanipulators. The sample was initially purged with dry nitrogen (200 sccm) for $90 \mathrm{~min}$. Resistance measurements were performed using an Agilent 34401A multimeter. During the initial nitrogen purge the resistance increased above the upper detection limit of the instrument. After the initial drying step the sensor was exposed to $25 \%$ $\mathrm{RH}\left(\right.$ at $\left.30{ }^{\circ} \mathrm{C}\right)$ in nitrogen $(200 \mathrm{sccm})$ until the resistance dropped below $1 \mathrm{G} \Omega$. The sample was then exposed to gas flows $(200 \mathrm{sccm})$ of specified moisture contents (between $25 \%$ and $60 \% \mathrm{RH}\left(\right.$ at $30{ }^{\circ} \mathrm{C}$ )) in nitrogen. A V-Gen Dew Point/RH generator was used to generate specific probe gas streams with $\mathrm{RH}$ values measured at $30{ }^{\circ} \mathrm{C}$. The sealed measurement chamber was also set to $30^{\circ} \mathrm{C}$. Photoconductivity measurements and characterizations were carried out using an identical device to that employed for the moisture sensing. Instead of exposing the sample to specific gas mixtures, the experiment was conducted in ambient atmosphere ( $\left.\mathrm{RH} 53 \%, 20^{\circ} \mathrm{C}\right)$. XRD measurements were conducted on a Bruker D4 Endeavor powder diffractometer with $\mathrm{Cu}$ $\mathrm{K} \alpha$ radiation of $1.5406 \AA$. The dried sample was stored for $100 \mathrm{~h}$ in a nitrogen glovebox prior to the analysis. The dried sample was sealed under a layer of Kapton film (TF-475), in a specifically designed sample holder for dry measurements. For consistency the ambient atmosphere dried sample was also measured under the Kapton film. A diffraction pattern of the Kapton film on its own was measured and is presented in the Supporting Information (Figure SI-14). Photoelectron spectroscopy in air was conducted using a Riken Keiki AC-2 photoelectron spectrometer. The samples were prepared by drop casting suspensions onto a microscopy glass slide. Mott-Schottky analysis was conducted using a $\mathrm{CH}$ Instruments $\mathrm{C} 600$ electrochemical workstation (potentiostat) equipped with an impedance module. A $\mathrm{Ag} / \mathrm{AgCl}$ reference electrode and a carbon rod counter electrode were used. The working electrodes were prepared by drop casting a suspension of either $\mathrm{TiO}_{2}$ of $\mathrm{MoS}_{3{ }^{2} / 3}$ onto carbon paper. The measurements were conducted using a sinusoidal signal with an amplitude of $5 \mathrm{mV}$ and a frequency of $1 \mathrm{kHz}$. The active area was approximately $1 \mathrm{~cm}^{2}$. The electrolyte contained $0.1 \mathrm{M}$ tris buffer $\mathrm{pH}$ 8.1 and $0.1 \mathrm{M} \mathrm{KCl}$ as a supporting electrolyte.

\section{ASSOCIATED CONTENT}

\section{S Supporting Information}

The Supporting Information is available free of charge on the ACS Publications website at DOI: 10.1021/acsnano.7b01632.

Additional Raman spectroscopy data, XRD patterns, TEM images, FTIR data, tabulated NMR relaxation times, moisture-sensing data, PESA results, MottSchottky plots, photoconductivity data, photocatalytic film temperature data, additional water splitting data, and the schematic of the used sensor template (PDF)

\section{AUTHOR INFORMATION}

\section{Corresponding Authors}

*E-mail: Torben.Daeneke@RMIT.edu.au.

*E-mail: Kourosh.Kalantar@RMIT.edu.au.

ORCID

Torben Daeneke: 0000-0003-1142-8646

Michael S. Strano: 0000-0003-2944-808X

Kourosh Kalantar-zadeh: 0000-0001-6109-132X

\section{Author Contributions}

${ }^{\perp}$ T. Daeneke and N. Dahr contributed equally.

Notes

The authors declare no competing financial interest.

\section{ACKNOWLEDGMENTS}

We would like to thank Dr. Anthony Chesman from the CSIRO Nanomaterials and Devices Team for providing access to PESA. T.D. acknowledges RMIT University for the provision of funds through the Vice Chancellor's Research Fellow program. We would furthermore like to acknowledge funds received form the Australian Research Council (ARC-DP 140100170 and DE160100715). We also acknowledge the MNRF and RMMF facilities for providing access to equipment and resources.

\section{REFERENCES}

(1) Hisatomi, T.; Kubota, J.; Domen, K. Recent Advances in Semiconductors for Photocatalytic and Photoelectrochemical Water Splitting. Chem. Soc. Rev. 2014, 43, 7520-7535.

(2) Ran, J.; Zhang, J.; Yu, J.; Jaroniec, M.; Qiao, S. Z. Earth-Abundant Cocatalysts for Semiconductor-Based Photocatalytic Water Splitting. Chem. Soc. Rev. 2014, 43, 7787-7812.

(3) Ronge, J.; Deng, S.; Pulinthanathu Sree, S.; Bosserez, T.; Verbruggen, S. W.; Kumar Singh, N.; Dendooven, J.; Roeffaers, M. B. J.; Taulelle, F.; De Volder, M.; Detavernier, C.; Martens, J. A. AirBased Photoelectrochemical Cell Capturing Water Molecules from Ambient Air for Hydrogen Production. RSC Adv. 2014, 4, 2928629290.

(4) Spurgeon, J. M.; Lewis, N. S. Proton Exchange Membrane Electrolysis Sustained by Water Vapor. Energy Environ. Sci. 2011, 4, 2993-2998.

(5) Dionigi, F.; Vesborg, P. C. K.; Pedersen, T.; Hansen, O.; Dahl, S.; Xiong, A.; Maeda, K.; Domen, K.; Chorkendorff, I. Gas Phase Photocatalytic Water Splitting with $\mathrm{Rh}_{2-\mathrm{Y}} \mathrm{Cr}_{\mathrm{y}} \mathrm{O}_{3} / \mathrm{GaN}: \mathrm{ZnO}$ in $\mu$ Reactors. Energy Environ. Sci. 2011, 4, 2937-2942.

(6) Modestino, M. A.; Dumortier, M.; Hosseini Hashemi, S. M.; Haussener, S.; Moser, C.; Psaltis, D. Vapor-Fed Microfluidic Hydrogen Generator. Lab Chip 2015, 15, 2287-2296.

(7) Silva, C. G.; Corma, A.; Garcia, H. Metal-Organic Frameworks as Semiconductors. J. Mater. Chem. 2010, 20, 3141-3156.

(8) Hill, C. L.; Prosser-McCartha, C. M. Homogeneous Catalysis by Transition Metal Oxygen Anion Clusters. Coord. Chem. Rev. 1995, 143, 407-455.

(9) Hong, W. T.; Risch, M.; Stoerzinger, K. A.; Grimaud, A.; Suntivich, J.; Shao-Horn, Y. Toward the Rational Design of NonPrecious Transition Metal Oxides for Oxygen Electrocatalysis. Energy Environ. Sci. 2015, 8, 1404-1427.

(10) Chia, X.; Eng, A. Y. S.; Ambrosi, A.; Tan, S. M.; Pumera, M. Electrochemistry of Nanostructured Layered Transition-Metal Dichalcogenides. Chem. Rev. 2015, 115, 11941-11966.

(11) Tran, P. D.; Tran, T. V.; Orio, M.; Torelli, S.; Truong, Q. D.; Nayuki, K.; Sasaki, Y.; Chiam, S. Y.; Yi, R.; Honma, I.; Barber, J.; Artero, V. Coordination Polymer Structure and Revisited Hydrogen Evolution Catalytic Mechanism for Amorphous Molybdenum Sulfide. Nat. Mater. 2016, 15, 640-646. 
(12) Ting, L. R. L.; Deng, Y.; Ma, L.; Zhang, Y.-J.; Peterson, A. A.; Yeo, B. S. Catalytic Activities of Sulfur Atoms in Amorphous Molybdenum Sulfide for the Electrochemical Hydrogen Evolution Reaction. ACS Catal. 2016, 6, 861-867.

(13) Afanasiev, P.; Jobic, H.; Lorentz, C.; Leverd, P.; Mastubayashi, N.; Piccolo, L.; Vrinat, M. Low-Temperature Hydrogen Interaction with Amorphous Molybdenum Sulfides $\mathrm{MoS}_{\mathrm{x}}$. J. Phys. Chem. C 2009, 113, 4139-4146.

(14) Afanasiev, P. Synthesis of Finely Divided Molybdenum Sulfide Nanoparticles in Propylene Carbonate Solution. J. Solid State Chem. 2014, 213, 158-164.

(15) Ge, X.; Chen, L.; Zhang, L.; Wen, Y.; Hirata, A.; Chen, M. Nanoporous Metal Enhanced Catalytic Activities of Amorphous Molybdenum Sulfide for High-Efficiency Hydrogen Production. Adv. Mater. 2014, 26, 3100-3104.

(16) Afanasiev, P.; Bezverkhy, I. Synthesis of $\operatorname{MoS}_{\mathrm{x}}(5>\mathrm{X}>6)$ Amorphous Sulfides and Their Use for Preparation of $\mathrm{MoS}_{2}$ Monodispersed Microspheres. Chem. Mater. 2002, 14, 2826-2830.

(17) Huang, Z.; Luo, W.; Ma, L.; Yu, M.; Ren, X.; He, M.; Polen, S.; Click, K.; Garrett, B.; Lu, J.; Amine, K.; Hadad, C.; Chen, W.; Asthagiri, A.; Wu, Y. Dimeric $\left[\mathrm{Mo}_{2} \mathrm{~S}_{12}\right]^{2-}$ Cluster: A Molecular Analogue of $\mathrm{MoS}_{2}$ Edges for Superior Hydrogen-Evolution Electrocatalysis. Angew. Chem., Int. Ed. 2015, 54, 15181-15185.

(18) Zou, X.; Zhang, Y. Noble Metal-Free Hydrogen Evolution Catalysts for Water Splitting. Chem. Soc. Rev. 2015, 44, 5148-5180.

(19) Benck, J. D.; Chen, Z.; Kuritzky, L. Y.; Forman, A. J.; Jaramillo, T. F. Amorphous Molybdenum Sulfide Catalysts for Electrochemical Hydrogen Production: Insights into the Origin of Their Catalytic Activity. ACS Catal. 2012, 2, 1916-1923.

(20) Morales-Guio, C. G.; Hu, X. Amorphous Molybdenum Sulfides as Hydrogen Evolution Catalysts. Acc. Chem. Res. 2014, 47, 26712681.

(21) Mohamad Latiff, N.; Wang, L.; Mayorga-Martinez, C. C.; Sofer, Z.; Fisher, A. C.; Pumera, M. Valence and Oxide Impurities in $\mathrm{MoS}_{2}$ and $\mathrm{WS}_{2}$ Dramatically Change Their Electrocatalytic Activity Towards Proton Reduction. Nanoscale 2016, 8, 16752-16760.

(22) Laursen, A. B.; Vesborg, P. C. K.; Chorkendorff, I. A HighPorosity Carbon Molybdenum Sulphide Composite with Enhanced Electrochemical Hydrogen Evolution and Stability. Chem. Commun. 2013, 49, 4965-4967.

(23) Kibsgaard, J.; Jaramillo, T. F.; Besenbacher, F. Building an Appropriate Active-Site Motif into a Hydrogen-Evolution Catalyst with Thiomolybdate $\left[\mathrm{Mo}_{3} \mathrm{~S}_{13}\right]^{2-}$ Clusters. Nat. Chem. 2014, 6, 248253.

(24) Merki, D.; Fierro, S.; Vrubel, H.; Hu, X. Amorphous Molybdenum Sulfide Films as Catalysts for Electrochemical Hydrogen Production in Water. Chem. Sci. 2011, 2, 1262-1267.

(25) Lee, S. C.; Benck, J. D.; Tsai, C.; Park, J.; Koh, A. L.; AbildPedersen, F.; Jaramillo, T. F.; Sinclair, R. Chemical and Phase Evolution of Amorphous Molybdenum Sulfide Catalysts for Electrochemical Hydrogen Production. ACS Nano 2016, 10, 624-632.

(26) Lassalle-Kaiser, B.; Merki, D.; Vrubel, H.; Gul, S.; Yachandra, V. K.; Hu, X.; Yano, J. Evidence from in Situ X-Ray Absorption Spectroscopy for the Involvement of Terminal Disulfide in the Reduction of Protons by an Amorphous Molybdenum Sulfide Electrocatalyst. J. Am. Chem. Soc. 2015, 137, 314-321.

(27) Li, Y.; Yu, Y.; Huang, Y.; Nielsen, R. A.; Goddard, W. A.; Li, Y.; Cao, L. Engineering the Composition and Crystallinity of Molybdenum Sulfide for High-Performance Electrocatalytic Hydrogen Evolution. ACS Catal. 2015, 5, 448-455.

(28) Bhattacharya, R. N.; Lee, C. Y.; Pollak, F. H.; Schleich, D. M. Optical Study of Amorphous $\mathrm{MoS}_{3}$ : Determination of the Fundamental Energy Gap. J. Non-Cryst. Solids 1987, 91, 235-242.

(29) Shin, S.; Jin, Z.; Kwon, D. H.; Bose, R.; Min, Y.-S. High Turnover Frequency of Hydrogen Evolution Reaction on Amorphous $\mathrm{MoS}_{2}$ Thin Film Directly Grown by Atomic Layer Deposition. Langmuir 2015, 31, 1196-1202.

(30) Kwon, D. H.; Jin, Z.; Shin, S.; Lee, W.-S.; Min, Y.-S. A Comprehensive Study on Atomic Layer Deposition of Molybdenum
Sulfide for Electrochemical Hydrogen Evolution. Nanoscale 2016, 8, $7180-7188$.

(31) Tang, M. L.; Grauer, D. C.; Lassalle-Kaiser, B.; Yachandra, V. K.; Amirav, L.; Long, J. R.; Yano, J.; Alivisatos, A. P. Structural and Electronic Study of an Amorphous $\mathrm{MoS}_{3}$ Hydrogen-Generation Catalyst on a Quantum-Controlled Photosensitizer. Angew. Chem., Int. Ed. 2011, 50, 10203-10207.

(32) Bourgeteau, T.; Tondelier, D.; Geffroy, B.; Brisse, R.; LabertyRobert, C.; Campidelli, S.; de Bettignies, R.; Artero, V.; Palacin, S.; Jousselme, B. A $\mathrm{H}_{2}$-Evolving Photocathode Based on Direct Sensitization of $\mathrm{MoS}_{3}$ with an Organic Photovoltaic Cell. Energy Environ. Sci. 2013, 6, 2706-2713.

(33) Vrubel, H.; Hu, X. Growth and Activation of an Amorphous Molybdenum Sulfide Hydrogen Evolving Catalyst. ACS Catal. 2013, 3, 2002-2011.

(34) Morales-Guio, C. G.; Tilley, S. D.; Vrubel, H.; Grätzel, M.; Hu, X. Hydrogen Evolution from a Copper(I) Oxide Photocathode Coated with an Amorphous Molybdenum Sulphide Catalyst. Nat. Commun. 2014, 5, 3059.

(35) Zhang, W.; Zhou, T.; Zheng, J.; Hong, J.; Pan, Y.; Xu, R. WaterSoluble $\mathrm{MoS}_{3}$ Nanoparticles for Photocatalytic $\mathrm{H}_{2}$ Evolution. ChemSusChem 2015, 8, 1464-1471.

(36) Redman, D. W.; Kim, H. J.; Stevenson, K. J.; Rose, M. J. PhotoAssisted Electrodeposition of $\mathrm{MoS}_{\mathrm{x}}$ from Ionic Liquids on OrganicFunctionalized Silicon Photoelectrodes for $\mathrm{H}_{2}$ Generation. J. Mater. Chem. A 2016, 4, 7027-7035.

(37) Morozan, A.; Bourgeteau, T.; Tondelier, D.; Geffroy, B.; Jousselme, B.; Artero, V. Noble Metal-Free Hydrogen-Evolving Photocathodes Based on Small Molecule Organic Semiconductors. Nanotechnology 2016, 27, 355401.

(38) Seger, B.; Laursen, A. B.; Vesborg, P. C. K.; Pedersen, T.; Hansen, O.; Dahl, S.; Chorkendorff, I. Hydrogen Production Using a Molybdenum Sulfide Catalyst on a Titanium-Protected $\mathrm{n}+\mathrm{p}$-Silicon Photocathode. Angew. Chem., Int. Ed. 2012, 51, 9128-9131.

(39) Li, F.; Zhao, S.-F.; Chen, L.; Khan, A.; MacFarlane, D. R.; Zhang, J. Polyethylenimine Promoted Electrocatalytic Reduction of $\mathrm{CO}_{2}$ to $\mathrm{CO}$ in Aqueous Medium by Graphene-Supported Amorphous Molybdenum Sulphide. Energy Environ. Sci. 2016, 9, 216-223.

(40) Subrahmanyam, K. S.; Malliakas, C. D.; Sarma, D.; Armatas, G. S.; Wu, J.; Kanatzidis, M. G. Ion-Exchangeable Molybdenum Sulfide Porous Chalcogel: Gas Adsorption and Capture of Iodine and Mercury. J. Am. Chem. Soc. 2015, 137, 13943-13948.

(41) Balasingam, S. K.; Thirumurugan, A.; Lee, J. S.; Jun, Y. Amorphous $\mathrm{MoS}_{\mathrm{x}}$ Thin-Film-Coated Carbon Fiber Paper as a 3D Electrode for Long Cycle Life Symmetric Supercapacitors. Nanoscale 2016, 8, 11787-11791.

(42) Matsuyama, T.; Hayashi, A.; Ozaki, T.; Mori, S.; Tatsumisago, M. Electrochemical Properties of All-Solid-State Lithium Batteries with Amorphous $\mathrm{MoS}_{3}$ Electrodes Prepared by Mechanical Milling. J. Mater. Chem. A 2015, 3, 14142-14147.

(43) Traversa, E. Ceramic Sensors for Humidity Detection: The State-of-the-Art and Future Developments. Sens. Actuators, B 1995, 23, $135-156$.

(44) Chen, Z.; Lu, C. Humidity Sensors: A Review of Materials and Mechanisms. Sens. Lett. 2005, 3, 274-295.

(45) Tu, R.; Liu, X.-H.; Jiang, Y. Lowering the Regeneration Temperature of a Rotary Wheel Dehumidification System Using Exergy Analysis. Energy Convers. Manage. 2015, 89, 162-174.

(46) Golubovic, M. N.; Hettiarachchi, H. D. M.; Worek, W. M. Sorption Properties for Different Types of Molecular Sieve and Their Influence on Optimum Dehumidification Performance of Desiccant Wheels. Int. J. Heat Mass Transfer 2006, 49, 2802-2809.

(47) Ng, K. C.; Chua, H. T.; Chung, C. Y.; Loke, C. H.; Kashiwagi, T.; Akisawa, A.; Saha, B. B. Experimental Investigation of the Silica Gel-Water Adsorption Isotherm Characteristics. Appl. Therm. Eng. 2001, 21, 1631-1642.

(48) White, S. D.; Goldsworthy, M.; Reece, R.; Spillmann, T.; Gorur, A.; Lee, D.-Y. Characterization of Desiccant Wheels with Alternative 
Materials at Low Regeneration Temperatures. Int. J. Refrig. 2011, 34, $1786-1791$.

(49) Nguyen, D. N.; Nguyen, L. N.; Nguyen, P. D.; Thu, T. V.; Nguyen, A. D.; Tran, P. D. Crystallization of Amorphous Molybdenum Sulfide Induced by Electron or Laser Beam and Its Effect on $\mathrm{H}_{2}$-Evolving Activities. J. Phys. Chem. C 2016, 120, 2878928794.

(50) $\left(\mathrm{NH}_{4}\right)_{2}\left[\mathrm{Mo}_{3} \mathrm{~S}_{13}\right] \cdot \mathrm{H}_{2} \mathrm{O}\left(\left[\mathrm{NH}_{4}\right]_{2} \mathrm{Mo}_{3} \mathrm{~S}_{13}\left[\mathrm{H}_{2} \mathrm{O}\right]\right)$ Crystal Structure: Datasheet from "Pauling File Multinaries ed.-2012" in Springermaterials (Http://Materials.Springer.Com/Isp/ Crystallographic/Docs/Sd_1709709), Springer-Verlag Berlin Heidelberg \& Material Phases Data System (MPDS), Switzerland \& National Institute for Materials Science (NIMS), Japan.

(51) Chan, K. C.; Chao, C. Y. H.; Sze-To, G. N.; Hui, K. S. Performance Predictions for a New Zeolite $13 \mathrm{X} / \mathrm{CaCl}_{2}$ Composite Adsorbent for Adsorption Cooling Systems. Int. J. Heat Mass Transfer 2012, 55, 3214-3224.

(52) Fleury, M.; Kohler, E.; Norrant, F.; Gautier, S.; M’Hamdi, J.; Barré, L. Characterization and Quantification of Water in Smectites with Low-Field NMR. J. Phys. Chem. C 2013, 117, 4551-4560.

(53) Carr, H. Y.; Purcell, E. M. Effects of Diffusion on Free Precession in Nuclear Magnetic Resonance Experiments. Phys. Rev. 1954, 94, 630-638.

(54) Meiboom, S.; Gill, D. Modified Spin-Echo Method for Measuring Nuclear Relaxation Times. Rev. Sci. Instrum. 1958, 29, 688-691.

(55) Chang, C. H.; Chan, S. S. Infrared and Raman Studies of Amorphous $\mathrm{MoS}_{3}$ and Poorly Crystalline $\mathrm{MoS}_{2}$. J. Catal. 1981, 72, 139-148.

(56) Aliyev, A. S.; Elrouby, M.; Cafarova, S. F. Electrochemical Synthesis of Molybdenum Sulfide Semiconductor. Mater. Sci. Semicond. Process. 2015, 32, 31-39.

(57) Bandara, J.; Divarathne, C. M.; Nanayakkara, S. D. Fabrication of $n-p$ Junction Electrodes Made of $n$-Type $\mathrm{SnO}_{2}$ and $p$-Type $\mathrm{NiO}$ for Control of Charge Recombination in Dye Sensitized Solar Cells. Sol. Energy Mater. Sol. Cells 2004, 81, 429-437.

(58) Li, L.; Lu, N.; Liu, M. Effect of Dipole Layer on the Density-ofStates and Charge Transport in Organic Thin Film Transistors. Appl. Phys. Lett. 2013, 103, 253303.

(59) Bi, H.; Yin, K.; Xie, X.; Ji, J.; Wan, S.; Sun, L.; Terrones, M.; Dresselhaus, M. S. Ultrahigh Humidity Sensitivity of Graphene Oxide. Sci. Rep. 2013, 3, 2714.

(60) Tripathy, A.; Pramanik, S.; Cho, J.; Santhosh, J.; Abu Osman, N. Role of Morphological Structure, Doping, and Coating of Different Materials in the Sensing Characteristics of Humidity Sensors. Sensors 2014, 14, 16343.

(61) Leonard, K. C.; Nam, K. M.; Lee, H. C.; Kang, S. H.; Park, H. S.; Bard, A. J. $\mathrm{ZnWO}_{4} / \mathrm{WO}_{3}$ Composite for Improving Photoelectrochemical Water Oxidation. J. Phys. Chem. C 2013, 117, 15901-15910.

(62) Toh, R. J.; Sofer, Z.; Pumera, M. Transition Metal Oxides for the Oxygen Reduction Reaction: Influence of the Oxidation States of the Metal and Its Position on the Periodic Table. ChemPhysChem 2015, 16, 3527-3531.

(63) Daeneke, T.; Gräf, K.; Watkins, S. E.; Thelakkat, M.; Bach, U. Infrared Sensitizers in Titania-Based Dye-Sensitized Solar Cells Using a Dimethylferrocene Electrolyte. ChemSusChem 2013, 6, 2056-2060. (64) Salvador, P. Hole Diffusion Length in $\mathrm{n}-\mathrm{TiO}_{2}$ Single Crystals and Sintered Electrodes: Photoelectrochemical Determination and Comparative Analysis. J. Appl. Phys. 1984, 55, 2977-2985.

(65) Leng, W. H.; Barnes, P. R. F.; Juozapavicius, M.; O’Regan, B. C.; Durrant, J. R. Electron Diffusion Length in Mesoporous Nanocrystalline $\mathrm{TiO}_{2}$ Photoelectrodes During Water Oxidation. J. Phys. Chem. Lett. 2010, 1, 967-972.

(66) Yu, J.; Qi, L.; Jaroniec, M. Hydrogen Production by Photocatalytic Water Splitting over $\mathrm{Pt} / \mathrm{TiO}_{2}$ Nanosheets with Exposed (001) Facets. J. Phys. Chem. C 2010, 114, 13118-13125.

(67) Atkin, P.; Daeneke, T.; Wang, Y.; Carey, B. J.; Berean, K. J.; Clark, R. M.; Ou, J. Z.; Trinchi, A.; Cole, I. S.; Kalantar-zadeh, K. 2D
$\mathrm{WS}_{2} /$ Carbon Dot Hybrids with Enhanced Photocatalytic Activity. J. Mater. Chem. A 2016, 4, 13563-13571.

(68) Wang, L.; Sofer, Z.; Luxa, J.; Pumera, M. $\mathrm{Mo}_{\mathrm{x}} \mathrm{W}_{1-\mathrm{X}} \mathrm{S}_{2}$ Solid Solutions as 3D Electrodes for Hydrogen Evolution Reaction. Adv. Mater. Interfaces 2015, 2, 1500041.

(69) Scanlon, D. O.; Dunnill, C. W.; Buckeridge, J.; Shevlin, S. A.; Logsdail, A. J.; Woodley, S. M.; Catlow, C. R. A.; Powell, M. J.; Palgrave, R. G.; Parkin, I. P.; Watson, G. W.; Keal, T. W.; Sherwood, P.; Walsh, A.; Sokol, A. A. Band Alignment of Rutile and Anatase $\mathrm{TiO}_{2}$. Nat. Mater. 2013, 12, 798-801.

(70) Dionigi, F.; Vesborg, P. C. K.; Pedersen, T.; Hansen, O.; Dahl, S.; Xiong, A.; Maeda, K.; Domen, K.; Chorkendorff, I. Suppression of the Water Splitting Back Reaction on GaN:ZnO Photocatalysts Loaded with Core/Shell Cocatalysts, Investigated Using a $\mu$-Reactor. J. Catal. 2012, 292, 26-31.

(71) Kumari, S.; Turner White, R.; Kumar, B.; Spurgeon, J. M. Solar Hydrogen Production from Seawater Vapor Electrolysis. Energy Environ. Sci. 2016, 9, 1725-1733. 